\title{
CR-manifolds of dimension 5: A Lie algebra approach
}

\author{
Gregor Fels and Wilhelm Kaup
}

\begin{abstract}
We study real-analytic Levi degenerate hypersurfaces $M$ in complex manifolds of dimension 3, for which the CR-automorphism group Aut $(M)$ is a real Lie group acting transitively on $M$. We provide large classes of examples for such $M$, compute the corresponding groups Aut $(M)$ and determine the maximal subsets of $M$ that cannot be separated by global continuous CR-functions. It turns out that all our examples, although partly arising in different contexts, are locally CR-equivalent to the tube $\mathscr{T}=\mathscr{C} \times i \mathbb{R}^{3} \subset \mathbb{C}^{3}$ over the future light cone $\mathscr{C}:=\left\{x \in \mathbb{R}^{3}: x_{1}^{2}+x_{2}^{2}=x_{3}^{2}, x_{3}>0\right\}$ in 3 -dimensional space-time.
\end{abstract}

\section{Introduction}

The notion of a Cauchy-Riemann-manifold (CR-manifold for short) generalizes that of a complex manifold. While two complex manifolds of the same dimension are always locally equivalent, this is no longer true for CR-manifolds. A basic invariant for a CR-manifold is the socalled Levi form. In case this form is nondegenerate, CHERN and MOSER give in their celebrated paper [5] further invariants which characterize up to equivalence the local CR-structure of real hypersurfaces in $\mathbb{C}^{n+1}$. For hypersurfaces with degenerate Levi form the corresponding program seems to be much harder to overcome. Of course, in case the connected real-analytic (locally closed) hypersurface $M \subset \mathbb{C}^{n+1}$ has a Levi nondegenerate point, all points in a dense open subset of $M$ are of this type. Therefore, of a special interest are those hypersurfaces which have everywhere degenerate Levi form but are not Levi flat. Clearly, the smallest $n$ for which hypersurfaces $M \subset \mathbb{C}^{n+1}$ with this property can occur is $n=2$, that is, where $M$ has real dimension 5. In fact, the tube $\mathscr{T}:=\mathscr{C} \times i \mathbb{R}^{3} \subset \mathbb{C}^{3}$ over the future light cone $\mathscr{C}:=\left\{x \in \mathbb{R}^{3}\right.$ : $\left.x_{1}^{2}+x_{2}^{2}=x_{3}^{2}, x_{3}>0\right\}$ in 3 -dimensional space-time is a well known example of this type. This CR-manifold, which is the starting point for our studies, is even homogeneous in the sense that a Lie group of CR-automorphisms acts transitively. In a way, $\mathscr{T}$ may be considered as a model surface [7] for locally homogeneous Levi degenerate surfaces, similar to the sphere $S^{2 n+1} \subset \mathbb{C}^{n+1}$ for spherical surfaces in the Levi nondegenerate case, compare e.g. [4] for the classification of homogeneous surfaces of this type and [1] for general homogeneous CR-manifolds.

In this paper we examine several naturally occurring CR-manifolds of dimension 5 with degenerate Levi-form. Most (but not all) of these are locally homogeneous and 2-nondegenerate. Surprisingly, it turns out that all of the latter are locally equivalent to the tube $\mathscr{T}$ over the light cone. From the global point of view, however, there are large classes of pairwise CR-nonequivalent manifolds which all are locally CR-equivalent to the tube $\mathscr{T}$. The classification of all homogeneous CR-manifolds of this type can essentially be reduced to the study of homogeneous domains in the real projective space $\mathbb{P}_{3}(\mathbb{R})$.

The paper is organized as follows:

In Section 2 we fix notation and recall some basic facts concerning the geometry of CR-manifolds.

2000 Mathematics Subject Classification: 17C50, 32M15, 32M25, 32V10, 32V25. 
In Section 3 we recall some (more or less known) properties of the model example $\mathscr{T}$ and focus on an explicit description of the Lie algebra of all infinitesimal CR-automorphisms.

In Section 4 we investigate hypersurface germs at $0 \in \mathbb{C}^{3}$ which correspond to the partial normal form (A,ii,2) in [6] (the only one out of 8 for holomorphically nondegenerate hypersurfaces to which uniformly Levi degenerate hypersurfaces can belong). Besides the fact that a partial normal form presentation of a hypersurface germ $(M, 0)$ is not an invariant in the strict sense (since it still depends on the choice of an adapted coordinate system and a further group of local CR-automorphisms), in general, it is rather difficult to deduce from the information encoded in the partial normal form only whether $(M, 0)$ is locally homogeneous or not. In Proposition 4.9 we give for certain germs a criterion for local homogeneity. We then introduce a family $\left(\mathscr{M}_{t}\right)_{t \in \mathbb{R}}$ of germs belonging to the partial normal form (A,ii,2) and compute explicitly the Lie algebras $\mathfrak{h o l}\left(\mathscr{M}_{t}, 0\right)$ of of germs of infinitesimal CR-transformations at 0 . It turns out that $\left(\mathscr{M}_{t}\right)_{t \in \mathbb{R}}$ varies from $\mathfrak{s l}(2, \mathbb{R}) \times \mathfrak{s u}(2)(t<0)$ over a non semi-simple Lie algebra $(t=0)$ to $\mathfrak{s l}(2, \mathbb{R}) \times \mathfrak{s l}(2, \mathbb{R})$ $(t>0)$ and jumps for $t=1$ to the 10-dimensional Lie algebra $\mathfrak{s o}(2,3)$. As main result of the section we show that actually $\mathscr{M}_{1}$ is locally CR-equivalent to the tube $\mathscr{T}$ and provide an explicit local CR-isomorphism. Moreover, the family $\left(\mathscr{M}_{t}\right)_{t \in \mathbb{R}}$ helps to clarify several results which can be found in the literature, compare e.g. the concluding paragraph in Section 4.

In Section 5 we study global properties of CR-manifolds that are locally CR-equivalent to the tube $\mathscr{T}$. Our first result states that on the universal covering of $\mathscr{T}$, which is infinitely sheeted, every global continuous CR-function is the pull-back of a function defined on $\mathscr{T}$. We then introduce a CR-deformation family $\left(\mathscr{T}_{t}\right)_{t>0}$ of $\mathscr{T}$ with interesting properties: The $\mathscr{T}_{t}$ are pairwise CR-nonequivalent CR-manifolds locally CR-equivalent to $\mathscr{T}$ and $\mathscr{T}_{1}=\mathscr{T}$. On the other hand, all $\mathscr{T}_{t}$ are diffeomorphic to $\mathscr{T}$ as real manifolds. What concerns the separation properties by global CR-functions, the $\mathscr{T}_{t}$ behave differently for various $t$ : For instance, if $t=p / q$ with $p, q \in \mathbb{N}$ relatively prime, there are precisely $p$ points in $\mathscr{T}_{t}$ that cannot be separated from a given point in $\mathscr{T}_{t}$ by global CR-functions. Further, for $t$ irrational, there is a closed hypersurface $\mathscr{N}_{t} \subset \mathscr{T}_{t}$ such that every continuous CR-function on $\mathscr{T}_{t}$ is real-analytic outside $\mathscr{N}_{t}$, while there do exist continuous CR-functions on $\mathscr{T}_{t}$ which are not globally real-analytic.

In Section 6 we consider a hypersurface $\mathscr{R} \subset \mathbb{C}^{3}$ which, in a certain sense, is universal for all homogeneous CR-manifolds that are locally CR-equivalent to the tube $\mathscr{T}$. This hypersurface occurs as the smooth boundary part of the Lie ball in $\mathbb{C}^{3}$ (biholomorphic image of Siegel's upper halfplane in the space of symmetric $2 \times 2$-matrices via a Cayley transformation). One result is that every simply-connected homogeneous CR-manifold locally CR-equivalent to $\mathscr{T}$ is the universal covering of a domain in $\mathscr{R}$ on which a suitable subgroup of the CR-automorphism group Aut( $\mathscr{R})$ acts transitively. What concerns some natural group actions, we observe that the nonclosed orbits in the complex manifold $\operatorname{SL}(2, \mathbb{C})$ under the action of the group $\operatorname{SL}(2, \mathbb{R}) \times \operatorname{SL}(2, \mathbb{R})$ given by $z \mapsto g z h^{-1}$ or the nonclosed orbits in the same complex manifold, but under a different action, namely of $\operatorname{SL}(2, \mathbb{C}) \cong \operatorname{Spin}(1,3)$ acting by $z \mapsto g z \bar{g}^{\prime}$, are all CR-equivalent to certain homogeneous domains in $\mathscr{R}$.

We close by indicating in Section 7 how some results from Section 5 can be generalized to higher dimensions.

\section{Preliminaries}

In this paper we use essentially the same conventions and notation as in [11, section 2]: Let $Z$ be a complex manifold $Z$ and $\pi: T Z \rightarrow Z$ its tangent bundle. Then $T Z$ also has the structure of a complex manifold with $\pi$ being a holomorphic submersion. In particular, every tangent space $T_{a} Z, a \in Z$, is a complex vector space with $\operatorname{dim}_{\mathbb{C}} T_{a} Z=n$ if $Z$ has complex 
dimension $n$ at $a$. By $\mathfrak{h o r}(Z)$ we denote the complex Lie algebra of all holomorphic vector fields on $Z$, that is, of all holomorphic sections $\xi: Z \rightarrow T M$ in the tangent bundle over $Z$. For every $a \in Z$ we denote the corresponding tangent vector in $T_{a} Z$ with $\xi_{a}$ (and not with $\xi(a)$ ).

A (locally closed) real submanifold $M \subset Z$ is called a $C R$-manifold if the complex dimension of the holomorphic tangent space $H_{a} M:=T_{a} M \cap i T_{a} M$ is a locally constant function of $a \in M$ (here every tangent space $T_{a} M$ is considered as an $\mathbb{R}$-linear subspace of $T_{a} Z$ ). A smooth function $f: M \rightarrow \mathbb{C}$ is called $\mathrm{CR}$ if for every $a \in M$ the restriction of its differential to $H_{a} M$ is complex linear. More generally, a smooth mapping $\varphi: M \rightarrow M^{\prime}$ between CR-manifolds is CR, if the differential $d_{a} \varphi: T_{a} M \rightarrow T_{\varphi(a)} M^{\prime}$ maps $H_{a} M$ to $H_{\varphi(a)} M^{\prime}$ and is complex linear thereon. The notion of CR-function and CR-mapping can also be generalized to the nonsmooth case; then the conditions have to hold in the distribution sense, see [2] as a general reference for CR-manifolds.

In the following we only consider connected real-analytic CR-submanifolds $M$ of $Z$. With $\mathfrak{h o l}(M)$ we denote the real Lie algebra of all vector fields $\eta: M \rightarrow T M \subset T Z$ on $M$ with the following property: To every $a \in M$ there is an open neighbourhood $U$ of $a$ with respect to $Z$ and a holomorphic vector field $\xi \in \mathfrak{h o l}(U)$ with $\xi_{z}=\eta_{z}$ for all $z \in U \cap M$. Clearly, in case the CR-submanifold $M$ is generic in $Z$ (that is, satisfies $T_{a} Z=T_{a} M+i T_{a} M$ for every $a \in M)$, the local holomorphic extension $\xi$ above can always be chosen in such a way that $U$ is an open neighbourhood of all of $M$ in $Z$. The elements of $\mathfrak{h o l}(M)$ are also called infinitesimal $C R$-transformations on $M$, the reason being that the corresponding local flow on $M$ consists of real-analytic CR-transformations. The vector field $\eta \in \mathfrak{h o l}(M)$ is called complete on $M$ if the local flow actually consists of a one-parameter family $\left(g_{t}\right), t \in \mathbb{R}$, of global transformations. Then we write $\exp (\eta):=g_{1}$ and get a mapping exp : aut $(M) \rightarrow \operatorname{Aut}(M)$, where $\mathfrak{a u t}(M) \subset \mathfrak{h o l}(M)$ is the subset of all complete vector fields and $\operatorname{Aut}(M)$ is the group of all real-analytic CRdiffeomorphisms of $M$. In general, $\mathfrak{a} \mathfrak{u t}(M) \subset \mathfrak{h o l}(M)$ is not closed under addition nor under taking brackets. But in case there is a Lie subalgebra $\mathfrak{g} \subset \mathfrak{h o l}(M)$ of finite dimension containing $\mathfrak{a u t}(M)$, then a result of [14] implies: $\mathfrak{a u t}(M)$ itself is a Lie algebra of finite dimension and Aut $(M)$ has a unique Lie group structure such that $\exp : \mathfrak{a u t}(M) \rightarrow \operatorname{Aut}(M)$ is locally bianalytic in a neighbourhood of $0 \in \mathfrak{a u t}(M)$.

For every $a \in M$ denote by $\mathfrak{h o l}(M, a)$ the space of all germs at $a$ of infinitesimal CRtransformations defined in arbitrary open neighbourhoods of $a$. Then $\mathfrak{h o l}(M, a)$ is a real Lie algebra, and $\mathfrak{a u t}(M, a):=\left\{\xi \in \mathfrak{h o l}(M, a): \xi_{a}=0\right\}$ is a Lie subalgebra of finite codimension. We call the CR-manifold $M$ locally homogeneous if the evaluation map $\mathfrak{h o l}(M, a) \rightarrow T_{a} M$, $\xi \mapsto \xi_{a}$, is surjective for every $a \in M$. Local homogeneity implies that to every pair $a, b$ of points in $M$ there exist open neighbourhoods $U, V$ of $a, b$ in $M$ together with a real-analytic CR-diffeomorphism $U \rightarrow V$. We call $M$ homogeneous if there exists a connected Lie group $G$ together with a group homomorphism $\Phi: G \rightarrow$ Aut $(M)$ such that the mapping $G \times M \rightarrow M$, $(g, x) \mapsto \Phi(g) x$, is real-analytic and $G$ acts transitively on $M$ via $\Phi$. Clearly, 'homogeneous' implies 'locally homogeneous'.

The CR-manifold $M \subset Z$ is called holomorphically nondegenerate if for every domain $U \subset Z$ and every $\xi \in \mathfrak{h o l}(U)$ with $\xi_{x}, i \xi_{x} \in T_{x} M$ for all $x \in U \cap M$ necessarily $\xi_{x}=0$ holds for all $x \in U \cap M$. In case $M$ is a real-analytic hypersurface in $Z$, holomorphic nondegeneracy is equivalent to $\operatorname{dim} \mathfrak{h o l}(M, a)<\infty$ for all $a \in M$, see e.g. [2] p. 367 for this and related results. In this note (except in the final section 7 ) the complex manifold $Z$ always has dimension 3 (either $\mathbb{C}^{3}$ or a nonsingular quadric in $\mathbb{P}_{4}(\mathbb{C})$ ) and $M$ is a real hypersurface that is 2-nondegenerate, a property that implies 'holomorphically nondegenerate'.

Convention for notating vector fields. In this paper we do not need the complexified tangent bundle $T M \otimes_{\mathbb{R}} \mathbb{C}$ of $M$. All vector fields occurring here correspond to 'real vector fields' elsewhere. In particular, if $E$ is a complex vector space of finite dimension and $U \subset E$ is an open subset 
then the vector fields $\xi \in \mathfrak{h o l}(U)$ correspond to holomorphic mappings $f: U \rightarrow E$, and the correspondence is given in terms of the canonical trivialization $T U \cong U \times E$ by identifying the mapping $f$ with the vector field $\xi=\left(\mathrm{id}_{U}, f\right)$. To have a short notation we also write

$$
\xi=f(z) \partial / \partial z
$$

As an example, if $E$ is the space of all complex $n \times m$-matrices and $c$ is an $m \times n$-matrix, then $z c z \partial / \partial z$ denotes the quadratic vector field on $E$ corresponding to the holomorphic mapping $E \rightarrow$ $E, z \mapsto z c z$. As soon as the vector field $\xi=f(z) \partial / \partial z$ is considered as differential operator, special caution is necessary: $\xi$ applied to the smooth function $h$ on $U$ is $\xi h=f(z) \partial / \partial z h+\bar{f}(z) \partial / \partial \bar{z} h$. We therefore stress again that we write

$$
\xi=f(z) \partial / \partial z \quad \text { instead of } \quad \xi=f(z) \partial / \partial z+\bar{f}(z) \partial / \partial \bar{z} \quad \text { elsewhere }
$$

and this convention will be in effect allover the paper. In particular, in case $E=\mathbb{C}^{n}$ with coordinates $z=\left(z_{1}, \ldots, z_{n}\right)$ we write

$$
\xi=f_{1}(z) \partial / \partial z_{1}+f_{2}(z) \partial / \partial z_{2}+\ldots+f_{n}(z) \partial / \partial z_{n},
$$

where $f=\left(f_{1}, \ldots, f_{n}\right): U \rightarrow \mathbb{C}^{n}$ is holomorphic.

\section{The tube over the light cone}

In this section we introduce the light cone tube $\mathscr{T}$, a real hypersurface of $\mathbb{C}^{3}$ that is everywhere Levi degenerate but has finite dimensional Lie algebra $\mathfrak{h o l}(\mathscr{T}, a)$ at every point $a \in \mathscr{T}$. From [11] we recall the explicit form of this Lie algebra and present a root decomposition in terms of vector fields. This will enable us in the next section to show that a certain local equation defines a CR-manifold locally equivalent to $\mathscr{T}$.

A convenient model for 3-dimensional space time is the linear subspace $V \subset \mathbb{R}^{2 \times 2}$ of all real symmetric $2 \times 2$-matrices with the (normalized) trace as time coordinate. There

$$
\Omega:=\{v \in V: v \text { positive definite }\}
$$

is the future cone and its smooth boundary part is the future light cone

$$
\mathscr{C}:=\{v \in V: \operatorname{det}(v)=0, \operatorname{tr}(v)>0\}=\left\{\left(\begin{array}{cc}
t+x_{1} & x_{2} \\
x_{2} & t-x_{1}
\end{array}\right) \in V: t^{2}=x_{1}^{2}+x_{2}^{2}, t>0\right\} .
$$

It is obvious that there exists a 2-dimensional group of linear transformations on $\mathbb{R}^{3}$ acting simply transitive on $\mathscr{C}$.

The main object of our interest is the tube

$$
\mathscr{T}:=\mathscr{C} \oplus i V=\{z \in V \oplus i V: \operatorname{det}(z+\bar{z})=0, \operatorname{Re} \operatorname{tr}(z)>0\}
$$

over $\mathscr{C}$, where we identify the complexification $V \oplus i V$ in the obvious way with the space $E$ of all symmetric complex $2 \times 2$-matrices. $\mathscr{T}$ is the smooth boundary part of the tube domain $\mathscr{H}:=\Omega \oplus i V$ in $E$ (Siegel's upper half plane up to the factor $i$ ) and is a locally closed realanalytic hypersurface of $E$ with everywhere degenerate Levi form. Actually it is well known that $\mathscr{T}$ is everywhere 2-nondegenerate as CR-manifold. 
The CR-automorphisms. The 7-dimensional Lie group of affine transformations on $E$

$$
\left\{z \mapsto g z g^{\prime}+i v: g \in \mathrm{GL}(2, \mathbb{R}), v \in V\right\}
$$

acts transitively on $\mathscr{T}$ and $\mathscr{H}$, where $g^{\prime}$ denotes the transpose of $g$. It is known [11] that $\operatorname{Aut}(\mathscr{T})$ is the group of all transformations (3.2) while Aut $(\mathscr{H})$ is the 10-dimensional group of all biholomorphic transformations

$$
z \mapsto(a z-i b)(i c z+d)^{-1},
$$

where $\left(\begin{array}{ll}a & b \\ c & d\end{array}\right)$ is in the real symplectic subgroup $\operatorname{Sp}(2, \mathbb{R}) \subset \mathrm{SL}(4, \mathbb{R})$ with $a, b, c, d \in \mathbb{R}^{2 \times 2}$, see [12] p. 351. Differentiating the action of $\mathrm{Sp}(2, \mathbb{R})$ gives

$$
\mathfrak{g}:=\mathfrak{a u t}(\mathscr{H})=\left\{\left(b+c z+z c^{\prime}+z d z\right) \partial / \partial z: b, d \in i V, c \in \mathbb{R}^{2 \times 2}\right\} \cong \mathfrak{s p}(2, \mathbb{R}) \cong \mathfrak{s o}(2,3)
$$

with convention (2.1) in effect. All vector fields in $\mathfrak{a u t}(\mathscr{H})$ are polynomial of degree $\leq 2$ on $E$, in particular,

$$
\mathfrak{a u t}(\mathscr{H}) \subset \mathfrak{h o l}(\mathscr{T}) \subset \mathfrak{h o l}(\mathscr{T}, a)
$$

in a canonical way for all $a \in \mathscr{T}$. One of the main results of [11] states (even for higher dimensional examples of this type) that all three Lie algebras above coincide, see Proposition 4.3 in [11].

The vector fields in $\mathfrak{g}$ corresponding to $c=\left(\begin{array}{ll}1 & 0 \\ 0 & 0\end{array}\right),\left(\begin{array}{ll}0 & 0 \\ 0 & 1\end{array}\right)$ in $(3.4)$ are

$$
\zeta_{1}:=2 z_{0} \partial / \partial z_{0}+z_{1} \partial / \partial z_{1} \quad \text { and } \quad \zeta_{2}:=z_{1} \partial / \partial z_{1}+2 z_{2} \partial / \partial z_{2}
$$

when expressed in the coordinates

$$
\left(z_{0}, z_{1}, z_{2}\right) \longmapsto\left(\begin{array}{cc}
z_{0} & z_{1} \\
z_{1} & z_{2}
\end{array}\right)
$$

on $E$. These give the following decomposition

$$
\mathfrak{g}=\bigoplus_{\nu \in \mathbb{Z}^{2}} \mathfrak{g}^{\nu} \quad \text { with } \quad\left[\mathfrak{g}^{\mu}, \mathfrak{g}^{\nu}\right] \subset \mathfrak{g}^{\mu+\nu}
$$

where $\mathfrak{g}^{\nu}:=\left\{\xi \in \mathfrak{g}:\left[\zeta_{j}, \xi\right]=\nu_{j} \xi\right.$ for $\left.j=1,2\right\}$ for $\nu=\left(\nu_{1}, \nu_{2}\right)$ and, in particular, $\mathfrak{g}^{0}=$ $\mathbb{R} \zeta_{1} \oplus \mathbb{R} \zeta_{2}$. For every root (i.e. $\nu \neq 0$ and $\mathfrak{g}^{\nu} \neq 0$ ) the corresponding root space $\mathfrak{g}^{\nu}$ has real dimension 1, and the set of all roots is visualized by the eight vectors in Figure 1, the root system of the complex simple Lie algebra $\mathfrak{s o}(5, \mathbb{C}) \cong \mathfrak{s p}(2, \mathbb{C})$.

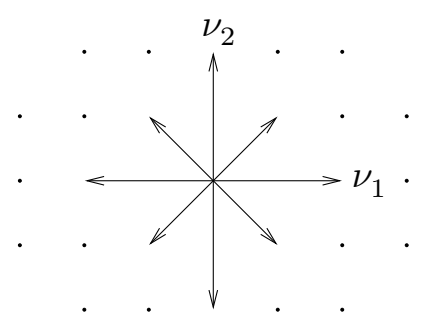

Figure 1

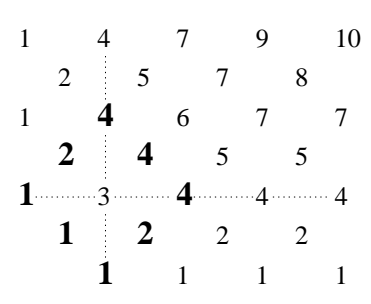

Figure 2 
An explicit choice of root vectors $\xi^{\nu} \in \mathfrak{g}^{\nu}$ is as follows:

$$
\begin{gathered}
\xi^{0,2}=i z_{1}^{2} \partial / \partial z_{0}+i z_{1} z_{2} \partial / \partial z_{1}+i z_{2}^{2} \partial / \partial z_{2} \\
\xi^{1,1}=2 i z_{0} z_{1} \partial / \partial z_{0}+i\left(z_{1}^{2}+z_{0} z_{2}\right) \partial / \partial z_{1}+2 i z_{1} z_{2} \partial / \partial z_{2} \\
\xi^{-1,1}=2 z_{1} \partial / \partial z_{0}+z_{2} \partial / \partial z_{1} \\
\xi^{-2,0}=i \partial / \partial z_{0}=i z_{0}^{2} \partial / \partial z_{0}+i z_{0} z_{1} \partial / \partial z_{1}+i z_{1}^{2} \partial / \partial z_{2} \\
\xi^{-1,-1}=i \partial / \partial z_{1} \\
\xi^{0,-2}=i \partial / \partial z_{2} \cdot
\end{gathered}
$$

Remarks. The choice of the vector fields (3.5) gives the Cartan subalgebra $\mathfrak{g}^{0}=\mathbb{R} \zeta_{1} \oplus \mathbb{R} \zeta_{2}$ of

$\mathfrak{g}$. Unlike to the complex situation there may exist several nonconjugate Cartan subalgebras in a real semi-simple Lie algebra. In our particular situation $\mathfrak{g} \cong \mathfrak{s o}(2,3)$ there are precisely four conjugacy classes of Cartan subalgebras.

Note that all vector fields in $\mathfrak{g}^{0}$ vanish simultaneously in a unique point of the nonsmooth boundary part $i V$ of $\mathscr{H}$, namely in the origin $0 \in E$. In Section 4 we will consider another Cartan subalgebra of $\mathfrak{g}$ that vanishes in a point of the smooth boundary part $\mathscr{T}$ of $\mathscr{H}$, and in Section 6 even a third one is considered that vanishes in a point of $\mathscr{H}$, compare (6.2).

It is not a coincidence that the Cartan subalgebras above vanish at some point of $E$. To put it in a broader perspective, $E$ admits a natural embedding in a compact complex flag manifold $Z$ together with a global action of $\operatorname{Sp}(2, \mathbb{R})$ on $Z$ that extends the action (3.3). By Borel's fixed point theorem every torus $H:=\exp (\mathfrak{h}), \mathfrak{h} \subset \mathfrak{g}$ a Cartan subalgebra, has a fixed point in $Z$.

The only nonclosed orbits in $E$ of the group

$$
H:=\left\{z \mapsto g z g^{\prime}+i v: g \in \mathrm{SL}(2, \mathbb{R}), v \in V\right\}
$$

are $\mathscr{T}$ and $-\mathscr{T}$. Furthermore, the real function $\operatorname{det}(z+\bar{z})$ is constant on $H$-orbits and its sign determines the signature of the Levi form for the orbits.

\section{A local realization}

In this section we introduce a family $\left(\mathscr{M}_{t}\right)_{t \in \mathbb{R}}$ of local CR-submanifolds in $\mathbb{C}^{3}$ that may be considered as local deformations of the tube $\mathscr{T}$, are pairwise CR-inequivalent and have semisimple Lie algebras $\mathfrak{h o l}\left(\mathscr{M}_{t}, 0\right), t \neq 0$, of dimension $\geq 6$. In particular, with $\mathscr{M}_{1}$ we get a normalized equation for $\mathscr{T}$ in the sense of [7].

In the following we consider in $\mathbb{C}^{3}$ with coordinates $\left(w, z_{1}, z_{2}\right)$ hypersurfaces $M$ of the following type. $M$ is given near $0 \in \mathbb{C}^{3}$ by an equation

$$
w+\bar{w}=2 z_{1} \bar{z}_{1}+\left(z_{1}^{2} \bar{z}_{2}+\bar{z}_{1}^{2} z_{2}\right)+q\left(\operatorname{Im}(w), z_{1}, \bar{z}_{1}, z_{2}, \bar{z}_{2}\right),
$$

where $q$ is a convergent real power series whose nonzero monomial terms either have degree $\geq 4$ or have degree 3 and then contain $\operatorname{Im}(w)$. Then (4.1) is just the partial normal form $(\mathrm{A}, \mathrm{ii}, 2)$ in [6]. In particular, $M$ is 2-nondegenerate at 0 and there the Levi kernel is the $z_{2}$-coordinate axis. Further, $\operatorname{dim} \mathfrak{h o l}(M, 0)<\infty$ holds.

Consider on $\mathbb{C}^{3}$ the group of all linear transformations $\left(w, z_{1}, z_{2}\right) \mapsto\left(s^{2} w, s t z_{1}, t^{2} z_{2}\right)$ with $s \in e^{\mathbb{R}}$ and $t \in e^{i \mathbb{R}}$. Clearly, this group is the exponential of the Lie algebra spanned in $\mathfrak{a u t}\left(\mathbb{C}^{3}\right)$ by the two (real) holomorphic vector fields $\zeta_{1}$ and $i \zeta_{2}$ where, using convention (2.1), $\zeta_{1}$ and $\zeta_{2}$ are given in the local coordinates by

$$
\zeta_{1}:=2 w \partial / \partial w+z_{1} \partial / \partial z_{1} \quad \text { and } \quad \zeta_{2}:=z_{1} \partial / \partial z_{1}+2 z_{2} \partial / \partial z_{2}
$$


The coordinate expression of these vector fields coincides with those in (3.5) (if the variable $w$ is renamed $z_{0}$ ). The same will occur in Section 6 .

From now on we assume for the rest of the section that $\mathfrak{g}:=\mathfrak{h} \mathfrak{o l}(M, 0)$ contains the vector fields $\zeta_{1}$ and $i \zeta_{2}$. For the sake of clarity let us emphasize that under this assumption the vector field $\zeta_{2}$ cannot be contained in $\mathfrak{g}$ since $M$ is holomorphically nondegenerate.

Our first goal is to show that under the assumption $\zeta_{1}, i \zeta_{2} \in \mathfrak{g}$ there is a unique equation of the form (4.1) such that $M$ is locally homogeneous at $0 \in M$. It will turn out that in this case $M$ is locally CR-equivalent to the tube $\mathscr{T}$ over the future light cone in $\mathbb{C}^{3}$ as considered in Section 2 .

We need some preparation: Let $\mathfrak{P}$ be the complex Lie algebra of all polynomial holomorphic vector fields on $\mathbb{C}^{3}$ with coordinates $w, z_{1}, z_{2}$. The natural adjoint action of $\zeta_{1}$ and $i \zeta_{2}$ induces a grading of $\mathfrak{P}$ : Consider the lattice

$$
\Lambda:=\{n+i m \in \mathbb{Z}+i \mathbb{Z}: n+m \in 2 \mathbb{Z}\}
$$

in $\mathbb{C}$ and denote by $\mathfrak{P}^{\lambda}$ the $\lambda$-eigenspace of $\operatorname{ad}\left(\zeta_{1}+i \zeta_{2}\right)$ in $\mathfrak{P}$. Note that every monomial vector field is contained in some $\mathfrak{P}^{\lambda}$ : For all $m, n, l \in \mathbb{N}$ and $k=0,1,2$ the vector field $w^{m} z_{1}^{n} z_{2}^{l} \partial / \partial z_{k}$ (with $\partial / \partial z_{0}:=\partial / \partial w$ to simplify notation) is in $\mathfrak{P}^{\lambda}$ for $\lambda=(2 m+n+k-2)+i(n+2 l-k)$. In particular, every $\mathfrak{P}^{\lambda}$ has finite dimension and

$$
\mathfrak{P}=\bigoplus_{\lambda \in \Lambda} \mathfrak{P}^{\lambda}, \quad\left[\mathfrak{P}^{\lambda}, \mathfrak{P}^{\mu}\right] \subset \mathfrak{P}^{\lambda+\mu}
$$

A small check shows that $\mathfrak{P}^{\lambda}=0$ if $\min (\operatorname{Re} \lambda, \operatorname{Im} \lambda, \operatorname{Re} \lambda+\operatorname{Im} \lambda)<-2$. For instance, for $\lambda \in$ $\{-2,-1-i,-2 i\}$ the spaces $\mathfrak{P}^{\lambda}=\mathbb{C} \xi^{\lambda}$ are 1-dimensional with generators

$$
\xi^{-2}:=i \partial / \partial w, \quad \xi^{-1-i}:=\partial / \partial z_{1}, \quad \xi^{-2 i}:=\partial / \partial z_{2} .
$$

In Figure 2 the nonzero complex dimensions of $\mathfrak{P}^{\lambda}$ are listed for all $\lambda=m+i n$ with $m \leq 6$ and $n \leq 4$.

We now explain how $\mathfrak{g}$ is related to the decomposition $\mathfrak{P}=\bigoplus \mathfrak{P}^{\lambda}$. A priori, the finite dimensional Lie algebra $\mathfrak{g}$ is contained in $\mathfrak{h o l}(U)$ for some open neighbourhood $U$ of $0 \in \mathbb{C}^{3}$. The sum $\mathfrak{l}:=\mathfrak{g}+i \mathfrak{g}$ in $\mathfrak{h o l}(U)$ actually is a direct sum of real subspaces. Since the complex Lie algebra $\mathfrak{l}$ contains the Euler vector field $\left(\zeta_{1}+\zeta_{2}\right) / 2$ necessarily $\mathfrak{l}$ is contained in $\mathfrak{P}$. Both $\mathfrak{l}$ and $\mathfrak{g}$ are invariant under $\operatorname{ad}\left(\zeta_{1}+i \zeta_{2}\right)$. This gives immediately the decomposition

$$
\mathfrak{l}=\bigoplus_{\lambda \in \Lambda} \mathfrak{l}^{\lambda}, \quad \mathfrak{l}^{\lambda}:=\mathfrak{l} \cap \mathfrak{P}^{\lambda} .
$$

Let $\xi \mapsto \bar{\xi}$ be the conjugation of $\mathfrak{l}$ with respect to the real form $\mathfrak{g}$. Then

$$
\overline{\mathfrak{l}^{\lambda}}=\mathfrak{l}^{\bar{\lambda}}
$$

for all $\lambda$ and consequently $\mathfrak{g} \cap \mathfrak{l}^{\lambda}=0$ if $\lambda \notin \mathbb{R}$. Furthermore, $\mathfrak{g}$ admits the generalized eigenspace decomposition

$$
\mathfrak{g}=\bigoplus_{\operatorname{Im} \lambda \geq 0} \mathfrak{g}^{[\lambda]}, \quad \mathfrak{g}^{[\lambda]}:=\mathfrak{g} \cap\left(\mathfrak{l}^{\lambda}+\mathfrak{l}^{\bar{\lambda}}\right)
$$


Next we claim that

$$
\mathfrak{g}^{[0]}=\mathbb{R} \zeta_{1} \oplus \mathbb{R} i \zeta_{2}
$$

holds. Indeed, assume to the contrary that there exists $\xi \in \mathfrak{g}^{[0]}$ with $\xi \notin\left(\mathbb{R} \zeta_{1}+\mathbb{R} i \zeta_{2}\right)$. Then $\xi \in \mathfrak{P}^{0}$ and, after subtracting a suitable linear combination of $\zeta_{1}, i \zeta_{2}$, we may assume $\xi=$ $\alpha w \partial / \partial w+\beta z_{2} \partial / \partial z_{2}$ for certain $\alpha, \beta \in \mathbb{C}$. Applying $\xi$ to the defining equation (4.1) yields on $M$ for $r:=\operatorname{Re}(\alpha)$ and $s:=\operatorname{Im}(\alpha)$ the identity

$$
r\left(2 z_{1} \bar{z}_{1}+z_{1}^{2} \bar{z}_{2}+\bar{z}_{1}^{2} z_{2}\right)-2 s \operatorname{Im}(w)=\beta \bar{z}_{1}^{2} z_{2}+\bar{\beta} z_{1}^{2} \bar{z}_{2}+\cdots
$$

up to terms of degree $\geq 4$ or of degree 3 containing $\operatorname{Im}(w)$ (the convention (2.1) has to be observed). This forces $\alpha=\beta=0$ and, in particular, $\xi \in\left(\mathbb{R} \zeta_{1}+\mathbb{R} i \zeta_{2}\right)$. 
Our first result now states

4.9 Proposition. Suppose that the $C R$-manifold $M$ is given locally by the equation (4.1) and that $\mathfrak{g}=$ $\mathfrak{h o l}(M, 0)$ contains the vector fields $\zeta_{1}, i \zeta_{2}$. Then the following conditions are equivalent.

(i) $M$ is locally homogeneous at 0.

(ii) $\mathfrak{g}$ contains the vector fields $\eta:=2 z_{1} \partial / \partial w+\left(1-z_{2}\right) \partial / \partial z_{1}$ and $\chi:=z_{1}^{2} \partial / \partial w-z_{1} z_{2} \partial / \partial z_{1}+\left(1-z_{2}^{2}\right) \partial / \partial z_{2}$.

(iii) The term $q$ in the defining equation (4.1) is

$$
q=\left(2 z_{1} \bar{z}_{1}+z_{1}^{2} \bar{z}_{2}+\bar{z}_{1}^{2} z_{2}\right) \cdot \sum_{j=1}^{\infty}\left(z_{2} \bar{z}_{2}\right)^{j}
$$

that is, the defining equation reads $w+\bar{w}=\left(2 z_{1} \bar{z}_{1}+z_{1}^{2} \bar{z}_{2}+\bar{z}_{1}^{2} z_{2}\right)\left(1-z_{2} \bar{z}_{2}\right)^{-1}$.

Proof. (i) $\Longrightarrow$ (ii) Suppose that $M$ is locally homogeneous at 0 . Then $\left\{\xi_{0}: \xi \in \mathfrak{l}\right\}=T_{0} M+$ $i T_{0} M \cong \mathbb{C}^{3}$. Since $\mathfrak{l}^{\lambda}$ vanishes at 0 for every $\lambda \notin\{-2,-1-i,-2 i\}$ we get $\operatorname{dim} \mathfrak{l}^{\lambda}=1$ for all $\lambda \in\{-2,-1 \pm i, \pm 2 i\}$ where we have used (4.6). Since every $\mathfrak{g}^{[\lambda]}$ is invariant under ad $\left(i \zeta_{2}\right)$ we have $\left\{\xi_{0}: \xi \in \mathfrak{g}^{[\lambda]}\right\}=\left\{\xi_{0}: \xi \in \mathfrak{l}^{\lambda}\right\}$ if $\operatorname{Im}(\lambda)<0$. Therefore there exists a unique $\xi \in \mathfrak{P}^{-1+i}$ with $\eta:=\xi^{-1-i}+\xi \in \mathfrak{g}$, that is, $\eta=\partial / \partial z_{1}+\alpha z_{1} \partial / \partial w+\beta z_{2} \partial / \partial z_{1} \in \mathfrak{g}$ for certain $\alpha, \beta \in \mathbb{C}$. Applying $\eta$ to the equation (4.1) and comparing homogeneous terms immediately gives $\alpha=2$ and $\beta=-1$. In the same way we get a vector field $\chi:=\partial / \partial z_{2}+z_{1}^{2} \partial / \partial w-\delta z_{1} z_{2} \partial / \partial z_{1}-\varepsilon z_{2}^{2} \partial / \partial z_{2} \in \mathfrak{g}^{[2 i]}$ for suitable $\delta, \varepsilon \in \mathbb{C}$. But then $\left[\mathfrak{g}^{[-1+i]}, \mathfrak{g}^{[2 i]}\right] \subset \mathfrak{g}^{[-1+i]}$ and

$$
[\eta, \chi]=\left(2 z_{1}+2(\delta-1) z_{1} z_{2}\right) \partial / \partial w+\left(1-\delta z_{2}+(\delta-\varepsilon) z_{2}^{2}\right) \partial / \partial z_{1} \in \mathfrak{g}^{[-1+i]},
$$

imply $[\eta, \chi]=\eta$, i.e. $\delta=\varepsilon=1$.

(ii) $\Longrightarrow$ (i) In case $\eta \in \mathfrak{g}^{[-1+i]}, \chi \in \mathfrak{g}^{[2 i]}$ the linear subspace $\mathfrak{g}^{[-1+i]} \oplus \mathfrak{g}^{[2 i]} \subset \mathfrak{g}$ spans the holomorphic tangent space $H_{0}(M) \subset T_{0} M$. The vector field $\left[\eta,\left[i \zeta_{2}, \eta\right]\right] \in \mathfrak{g}^{[-2]}$ does not vanish and thus spans $T_{0} M / H_{0} M$, that is, $\mathfrak{g}$ spans the full tangent space $T_{0} M$.

(iii) $\Longrightarrow$ (ii) This is easily checked.

(ii) $\Longrightarrow$ (iii) The $\mathbb{R}$-linear span of $\xi^{-2}, \eta, \chi,\left[i \zeta_{2}, \eta\right],\left[i \zeta_{2}, \chi\right], i \zeta_{2}$ is a Lie subalgebra $\mathfrak{a} \subset \mathfrak{g}$ of dimension 6 which also spans the full tangent space of $M$ at 0 . Therefore $M$ is the the local integral manifold of $\mathfrak{a}$ near $0 \in \mathbb{C}^{3}$. Denote by $\widetilde{M}$ the hypersurface defined near $0 \in \mathbb{C}^{3}$ by the equation (4.1), where $q$ is replaced by $\widetilde{q}=\left(2 z_{1} \bar{z}_{1}+z_{1}^{2} \bar{z}_{2}+\bar{z}_{1}^{2} z_{2}\right) \cdot \sum_{j=1}^{\infty}\left(z_{2} \bar{z}_{2}\right)^{j}$. By a routine check it is verified that also $\mathfrak{a} \subset \mathfrak{h o l}(\widetilde{M}, 0)$ holds, that is, also $\widetilde{M}$ is the local integral manifold of $\mathfrak{a}$ near $0 \in \mathbb{C}^{3}$. This implies that the germs of $M$ and $\widetilde{M}$ at 0 coincide and hence that $q=\widetilde{q}$ as power series.

A family of CR-manifolds. In the following we fix $t \in \mathbb{R}$ and consider the CR-hypersurface $\mathscr{M}_{t}$ given in $\mathbb{C}^{3}$ near the origin by the equation

$$
w+\bar{w}=\left(2 z_{1} \bar{z}_{1}+z_{1}^{2} \bar{z}_{2}+\bar{z}_{1}^{2} z_{2}\right)\left(1-t z_{2} \bar{z}_{2}\right)^{-1} .
$$

Clearly, this is a special case of (4.1), and it is easily checked that the vector fields $\zeta_{1}, i \zeta_{2}$ are contained in $\mathfrak{g}:=\mathfrak{g}_{t}:=\mathfrak{h o l}\left(\mathscr{M}_{t}, 0\right)$. The manifold $\mathscr{M}_{t}$ for $t=0$ has already been studied in [8] and for $t=1$ in [9], where also the vector fields $\eta, \chi$ from (4.9) occur.

Our next goal is to describe how the Lie algebra $\mathfrak{g}$ depends on the parameter $t$. Put $\Psi:=\{ \pm 2, \pm 2 i\}$ and define for each $\lambda \in \Psi \subset \Lambda$ the vector fields $\xi^{\lambda} \in \mathfrak{P}^{\lambda}$ as follows:

$$
\xi^{2 i}:=z_{1}^{2} \partial / \partial w-t z_{1} z_{2} \partial / \partial z_{1}-t z_{2}^{2} \partial / \partial z_{2}, \quad \xi^{2}:=i t w^{2} \partial / \partial w+i t w z_{1} \partial / \partial z_{1}-i z_{1}^{2} \partial / \partial z_{2}
$$

and $\xi^{-2 i}, \xi^{-2}$ as in (4.5). Furthermore put

$$
\eta^{\lambda}:= \begin{cases}\xi^{\lambda}+\xi^{\bar{\lambda}} & \operatorname{Im}(\lambda) \geq 0 \\ i \xi^{\lambda}-i \xi^{\bar{\lambda}} & \text { otherwise }\end{cases}
$$


and notice that

$$
\eta^{\lambda} \in \mathfrak{g}, \quad\left[\zeta_{1}, \eta^{\lambda}\right]=\operatorname{Re}(\lambda) \eta^{\lambda} \quad \text { and } \quad\left[i \zeta_{2}, \eta^{\lambda}\right]=\operatorname{Im}(\lambda) \eta^{\bar{\lambda}}
$$

hold for every $\lambda \in \Psi$. By applying vector fields to equation (4.10) it is not difficult to see that the Lie algebra

$$
\begin{aligned}
\mathfrak{h}: & =\left(\mathbb{R} \eta^{-2} \oplus \mathbb{R} \zeta_{1} \oplus \mathbb{R} \eta^{2}\right) \oplus\left(\mathbb{R} \eta^{-2 i} \oplus \mathbb{R} i \zeta_{2} \oplus \mathbb{R} \eta^{2 i}\right) \\
& \cong \begin{cases}\mathfrak{s l}(2, \mathbb{R}) \times \mathfrak{s l}(2, \mathbb{R}) & t>0 \\
\left(\mathfrak{s o}(1,1) \ltimes \mathbb{R}^{2}\right) \times\left(\mathfrak{s o}(2) \ltimes \mathbb{R}^{2}\right) & t=0 \\
\mathfrak{s l}(2, \mathbb{R}) \times \mathfrak{s u}(2) & t<0\end{cases}
\end{aligned}
$$

is contained in $\mathfrak{g}$. In case $t \neq 1$ the CR-manifold $\mathscr{M}_{t}$ cannot be locally homogeneous at 0 since then the vector field $\chi$ from (4.9) is not contained in $\mathfrak{g}^{[2 i]}=\mathbb{R} \eta^{2 i} \oplus \mathbb{R} \eta^{-2 i}$, compare Proposition 4.9. In case $t=0$ the right hand side of (4.10) reduces to a cubic polynomial and, with some computation, it can be seen that $\mathfrak{g}=\mathfrak{h}$ in this case, compare also case B2 in [8, p. 94]. In all other cases $\mathfrak{h}$ is semi-simple. We shall use this in the following.

Explicit determination of $\mathfrak{h o l}\left(\mathscr{M}_{t}, 0\right)$. For fixed $t \in \mathbb{R}$ and $\mathfrak{l}=\mathfrak{g} \oplus i \mathfrak{g}$ with $\mathfrak{g}=\mathfrak{h o l}\left(\mathscr{M}_{t}, 0\right)$ put

$$
\Phi:=\left\{\lambda \in \Lambda: \lambda \neq 0 \text { and } \mathfrak{l}^{\lambda} \neq 0\right\} .
$$

Then $\Psi \subset \Phi$ and $\Phi$ is invariant under $\lambda \mapsto \bar{\lambda}$. For every $k \in \mathbb{Z}$ denote by $d_{k}$ the dimension of the eigenspace $\left\{\xi \in \mathfrak{l}:\left[\zeta_{1}, \xi\right]=k \xi\right\}=\bigoplus_{\operatorname{Re} \lambda=k} \mathfrak{l}^{\lambda}$. In case $t \neq 0$ the representation theory of $\mathfrak{s l}(2, \mathbb{C}) \cong \mathbb{C} \eta^{-2} \oplus \mathbb{C} \zeta_{1} \oplus \mathbb{C} \eta^{2}$ implies $d_{k}=d_{-k}$ for all $k$, and thus $d_{1} \in\{0,2\}, d_{2}=1$ and $d_{k}=0$ for $k>2$. As a consequence, $\Phi$ is also invariant under $\lambda \mapsto-\bar{\lambda}$ and $\mathfrak{l}^{\lambda}$ has dimension 1 for every $\lambda \in \Phi$. Because of $(-1-i) \notin \Phi$ in case $t \neq 1$ we therefore have $\Phi=\Psi$ in this situation. This proves:

4.14 Proposition. In case $t \neq 1$ the Lie algebra $\mathfrak{h o l}\left(\mathscr{M}_{t}, 0\right)$ coincides with $\mathfrak{h}$ and, in particular, has dimension 6.

It remains to consider the case $t=1$. Applying $\eta$ to equation (4.10) implies $\eta \in \mathfrak{g}=$ $\mathfrak{h o l}\left(\mathscr{M}_{1}, 0\right)$. In particular $(-1-i) \in \Phi$ and hence $\Phi:=\{ \pm 2, \pm 1 \pm i, \pm 2 i\}$, that is, $\Phi$ can also be visualized by Figure 1, while $\Psi \subset \Phi$ corresponds to the subset of long arrows. Define for every $\lambda \in \Phi$ the vectors $\xi^{\lambda} \in \mathfrak{P}^{\lambda}$ by (4.5) and

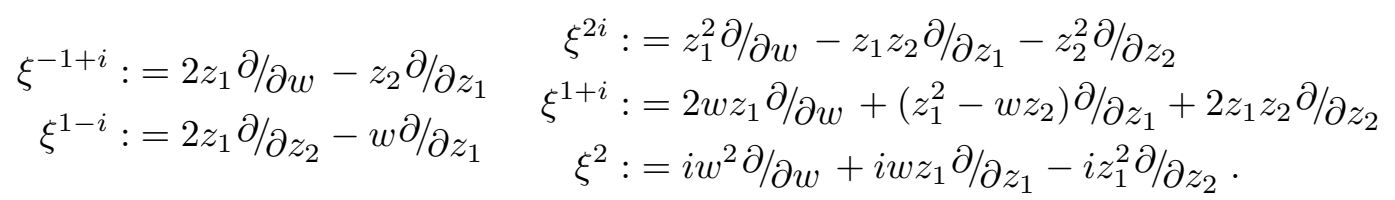

Notice that after replacing $z_{2}$ by $-z_{2}$ all vector fields in (4.15) and (4.5) become complex multiples of those in (3.7).

Now define $\eta^{\lambda}$ by (4.12) for every $\lambda \in \Phi$. Differentiating the defining equation for $M_{1}$ along $\eta^{\lambda}$ gives $\eta^{\lambda} \in \mathfrak{g}$ and hence $\mathfrak{l}^{\lambda}=\mathbb{C} \xi^{\lambda}$ for all $\lambda \in \Phi$. For all $\lambda, \mu \in \Phi$ with $\lambda+\mu \neq 0$ the identity $\left[\mathfrak{l}^{\lambda}, \mathfrak{l}^{\mu}\right]=\mathfrak{l}^{\lambda+\mu}$ can be verified, that is, $\mathfrak{l}$ is isomorphic to $\mathfrak{s o}(5, \mathbb{C})$. As real form of $\mathfrak{l}$ therefore $\mathfrak{g}$ is isomorphic to $\mathfrak{s o}(5), \mathfrak{s o}(1,4)$ or $\mathfrak{s o}(2,3)$. The isotropy subalgebra $\mathfrak{l}_{0}$ of $\mathfrak{l}$ at the origin of $\mathbb{C}^{3}$ is maximal parabolic. Therefore, $\mathscr{M}_{1}$ occurs as piece of an R-orbit in a 3 -dimensional quadric $Z \subset \mathbb{P}_{4}(\mathbb{C})$ or in $\mathbb{P}_{3}(\mathbb{C})$, where $\mathrm{R}$ is a real form of $\mathrm{SO}(5, \mathbb{C}) \cong \mathrm{Sp}(2, \mathbb{C})$. Since the only 2 -nondegenerate hypersurface orbits occur in case of $\mathrm{SO}(2,3)$ acting on $Z$, the Lie algebra $\mathfrak{g}$ can only be isomorphic to $\mathfrak{s o}(2,3)$. Since the tube $\mathscr{T}$ over the future light cone actually can be realized as an open piece of an $\mathrm{SO}(2,3)$-orbit in $Z$, we get (a direkt proof is given by formula (4.18)): 
4.16 Proposition. The hypersurface $\mathscr{M}_{1}$ defined near $0 \in \mathbb{C}^{3}$ by the equation

$$
w+\bar{w}=\left(2 z_{1} \bar{z}_{1}+z_{1}^{2} \bar{z}_{2}+\bar{z}_{1}^{2} z_{2}\right)\left(1-z_{2} \bar{z}_{2}\right)^{-1}
$$

is locally $C R$-isomorphic to the tube $\mathscr{T}$ over the future light cone.

The local CR-equivalence between $\mathscr{M}_{1}$ and $\mathscr{T}$ can be made explicit. For this, a transformation in $\mathrm{SO}(5, \mathbb{C})$ has to be found that the corresponding $\mathrm{SO}(2,3)$-orbits in the quadric $Z$ maps to each other. Let $U:=\left\{\left(w, z_{1}, z_{2}\right) \in \mathbb{C}^{3}:\left|z_{2}\right|<1\right\}$ and consider $\mathscr{M}_{1}$ as closed CR-submanifold of $U$ via the equation (4.17). Then

$$
\left(w, z_{1}, z_{2}\right) \longmapsto \frac{1}{1+z_{2}}\left(\begin{array}{cc}
w+w z_{2}+z_{1}^{2} & \sqrt{2} z_{1} \\
\sqrt{2} z_{1} & 1-z_{2}
\end{array}\right)
$$

defines a biholomorphic mapping $\varphi$ from $U$ to an open subset of $E$ with $\varphi\left(\mathscr{M}_{1}\right) \subset \mathscr{T}$. The inverse $\varphi^{-1}$ is given by

$$
\left(\begin{array}{ll}
x & y \\
y & t
\end{array}\right) \longmapsto \frac{1}{1+t}\left(x+x t-y^{2}, \sqrt{2} y, 1-t\right) .
$$

More generally, we consider $\mathscr{M}:=\mathscr{M}_{t}$ as a closed CR-hypersurface of $U_{t}:=\left\{\left(w, z_{1}, z_{2}\right) \in\right.$ $\left.\mathbb{C}^{3}: t z_{2} \bar{z}_{2}<1\right\}$ and $\mathfrak{g}=\mathfrak{h o l}(\mathscr{M}, 0)$ as a Lie algebra of holomorphic vector fields on $\mathbb{C}^{3}$. For fixed $t \neq 1$

$$
N:=\left\{\left(w, z_{1}, 0\right) \in \mathbb{C}^{3}: w=z_{1} \bar{z}_{1}\right\}
$$

is a transversal slice in $\mathscr{M}$ to the infinitesimal action of $\mathfrak{g}$ on $\mathscr{M}$. An elementary computation gives

$$
\left(\begin{array}{cc}
2 & 2 z_{1} \\
2 \bar{z}_{1} & 2 t z_{1} \bar{z}_{1}
\end{array}\right)
$$

for the Levi matrix, and hence $4(t-1) z_{1} \bar{z}_{1}$ for the Levi determinant at the point $\left(w, z_{1}, 0\right) \in N$. In particular, the infinitesimal $\mathfrak{g}$-orbit of the origin, that is $\left\{\left(w, 0, z_{2}\right) \in \mathbb{C}^{3}: w \in i \mathbb{R}, t z_{2} \bar{z}_{2}<1\right\}$, coincides with the set of all Levi degenerate points of $\mathscr{M}$. Furthermore, the Levi form at every point of $\mathscr{M} \backslash N$ is definite if $t>1$ and indefinite otherwise.

The family $\mathscr{M}_{t}$ yields counter examples to several statements found in the literature: For instance, contrary to Theorem 4 in [7], for every $\mathscr{M}_{t}$ the vector field $\xi^{2}=i t w^{2} \partial / \partial w+i t w z_{1} \partial / \partial z_{1}-$ $i z_{1}^{2} \partial / \partial z_{2} \in \mathfrak{h o l}\left(\mathscr{M}_{t}, 0\right)$ has vanishing 1 -jet at 0 . Furthermore, in [9] it is claimed that " $\mathscr{M}_{1}$ is a new example of a uniformly Levi-degenerate CR-manifold apart from $\mathscr{T}$," which cannot be true due to Proposition 4.16. Finally, translated to our notation, it is claimed in [8] p. 194 for the model surface $\mathscr{M}_{0}$ that " $\operatorname{dim} \mathfrak{h o l}(\mathscr{M}, 0) \leq \operatorname{dim} \mathfrak{h o l}\left(\mathscr{M}_{0}, 0\right)=6$ holds for every hypersurface $\mathscr{M}$ given by an equation of the form (4.1)". But for $\mathscr{M}_{1}$ this dimension is 10 .

\section{Global properties}

Let again $V, E, \mathscr{C}, \mathscr{T}, \mathscr{H}$ have the same meaning as in section 2 . The tube manifold $\mathscr{T}$ is a homogeneous 2-nondegenerate CR-manifold of dimension 5. In the following we want to present further CR manifolds of this type that are locally but not globally CR-equivalent to $\mathscr{T}$. 
Let us denote by $\widetilde{\mathscr{T}}$ the universal covering of $\mathscr{T}$ in the following and by $\mu: \widetilde{\mathscr{T}} \rightarrow \mathscr{T}$ the corresponding covering map. For convenience we identify $\widetilde{\mathscr{T}}$ with $\mathbb{R}^{2} \times V$ and $\mu$ with the mapping

$$
(r, \varphi, v) \longmapsto e^{r}\left(\begin{array}{cc}
1+\cos \varphi & \sin \varphi \\
\sin \varphi & 1-\cos \varphi
\end{array}\right)+i v, \quad r, \varphi \in \mathbb{R}, v \in V
$$

Clearly, the CR-structure on $\widetilde{\mathscr{T}}=\mathbb{R}^{2} \times V$ is uniquely determined by the condition that $\mu$ is a local CR-isomorphism.

Our first result states in particular that every continuous CR-function on $\widetilde{\mathscr{T}}$ is constant on $\mu$-fibers. For this denote by $\hat{\mathscr{T}}:=\mathscr{T} \cup \mathscr{H}$ the convex hull of $\mathscr{T}$ in $E$. Also, call for every open subset $U \subset \hat{\mathscr{T}}$ a continuous mapping $f: U \rightarrow \mathbb{C}^{n}, n \in \mathbb{N}$, holomorphic if its restriction to $U \cap \mathscr{H}$ is holomorphic in the usual sense.

5.2 Proposition. To every continuous CR-function $f$ on $\widetilde{T}$ there exists a unique holomorphic function $h$ on $\hat{\mathscr{T}}$ with $f=h \circ \mu$.

Proof. There exists a connected Lie group $G$ (for instance, the group of all transformations (3.2) with $\operatorname{det}(g)>0$ ) acting continuously on $\hat{\mathscr{T}}$ by biholomorphic transformations (in the extended sense defined above) such that $\mathscr{T}$ and $\mathscr{H}$ are $G$-orbits. We may assume that $G$ is simply connected, otherwise replace $G$ by its universal covering group. This guarantees that the action of $G$ on $\mathscr{T}$ lifts to a transitive action of $G$ on $\widetilde{\mathscr{T}}$. The sheaf $\mathcal{O}$ over $\hat{\mathscr{T}}$ of germs of local holomorphic functions (also in the extended sense) is a Hausdorff space for which the canonical projection $\pi: \mathcal{O} \rightarrow \hat{\mathscr{T}}$ is a local homeomorphism. Now fix an arbitrary point $a \in \mathscr{T}$ and choose an open neighbourhood $V$ of $a$ in $\mathscr{T}$. For $V$ small enough there exists a continuous section $\sigma: V \rightarrow \widetilde{\mathscr{T}}$, i.e. $\mu \circ \sigma=\left.\mathrm{id}\right|_{V}$. Since $\mathscr{T}$ is the smooth boundary part of the Stein domain $\mathscr{H}$, the Levi cone at $a \in \mathscr{T}$, (which is non zero) points into the direction of $\mathscr{H}$. Consequently, there exists a connected open neighbourhood $U$ of $a$ with respect to $\hat{\mathscr{T}}$ such that every continuous CR-function on $V$ has a holomorphic extension to $U$, compare [3] p. 256 or [2] p. 205. Fix now an arbitrary continuous CR-function $f$ on $\widetilde{T}$. For every $g \in G$ there exists a unique holomorphic function $f_{g}$ on $U$ which coincides with $f \circ g \circ \sigma$ on $U \cap V$. Denote by $\mathcal{F}_{g} \subset \mathcal{O}$ the subset of all germs induced by the holomorphic function $f_{g} \circ g^{-1}$, defined on $g(U)$. Clearly, every $\mathcal{F}_{g}$ is connected. Observe that for sufficiently close $g_{1}, g_{2} \in G$, the intersection $\mathcal{F}_{g_{1}} \cap \mathcal{F}_{g_{2}}$ is not empty. Since $G$ is connected, there is a unique connected component $\mathcal{F}$ of $\mathcal{O}$ containing all $\mathcal{F}_{g}, g \in G$. We claim that $\pi: \mathcal{F} \rightarrow \hat{\mathscr{T}}$ is a covering map. Since $\pi$ is a local homeomorphism it is sufficient to show that every continuous curve $\gamma:[0,1] \rightarrow \hat{\mathscr{T}}$ has a lifting to a continuous curve $\tau:[0,1] \rightarrow \mathcal{F}$ with $\pi \circ \tau=\gamma$. Without lost of generality we may consider only curves $\gamma$ in $\mathscr{H}$. The claim follows from the existence of a continuous curve $t \mapsto g_{t}$ in $G$ with $\gamma(t)=g_{t}(\gamma(0))$ for all $t \in[0,1]$. Since $\hat{\mathscr{T}}$ is simply connected we get that $\pi: \mathcal{F} \rightarrow \hat{\mathscr{T}}$ is a bijection. Therefore $\mathcal{F}$ is a continuous section in $\mathcal{O}$ over $\hat{\mathscr{T}}$ and determines the required holomorphic function $h$ on $\hat{\mathscr{T}}$.

5.3 Corollary. To every $\widetilde{g} \in \operatorname{Aut}(\widetilde{\mathscr{T}})$ there exists a unique $g \in \operatorname{Aut}(\mathscr{T})$ with $\mu \circ \widetilde{g}=g \circ \mu$. The mapping Aut $(\widetilde{\mathscr{T}}) \rightarrow \operatorname{Aut}(\mathscr{T}), \widetilde{g} \mapsto g$, realizes Aut $(\widetilde{\mathscr{T}})$ as universal covering group of Aut $(\mathscr{T})$. In particular, Aut $(\widetilde{T})$ is a simply connected Lie group of dimension 7 with two connected components, acting transitively on $\widetilde{T}$.

Proof. Denote by $\iota: \mathscr{T} \hookrightarrow E$ the canonical injection. By Proposition 5.2 the mapping $\iota \circ \mu \circ \widetilde{g}$ is constant on $\mu$-fibers and hence factors over $\mu$. The group Aut $(\mathscr{T})$ is given by all affine transformations (3.2) and hence there is a canonical isomorphism of Lie groups

$$
\operatorname{Aut}(\mathscr{T}) \cong \mathrm{GL}(\mathscr{C}) \ltimes V,
$$


where

$$
\mathrm{GL}(\mathscr{C}):=\{g \in \mathrm{GL}(V): g(\mathscr{C})=\mathscr{C}\} \cong(\mathrm{GL}(2, \mathbb{R}) /\{ \pm e\}),
$$

$e \in \mathrm{GL}(2, \mathbb{R})$ is the unit matrix and the semi-direct product refers to the canonical injection $\rho: \mathrm{GL}(\mathscr{C}) \hookrightarrow \mathrm{GL}(V)$. In particular, Aut $(\widetilde{\mathscr{T}}) \cong \widetilde{\mathrm{GL}}(\mathscr{C}) \ltimes V$ with $\widetilde{\mathrm{GL}}(\mathscr{C}) \cong \widetilde{\mathrm{GL}}(2, \mathbb{R})$ the universal covering group of $\mathrm{GL}(\mathscr{C})$.

A CR-deformation family for $\mathscr{T}$. For every $\psi \in \mathbb{R}$ and $\mu_{\psi}:=\left(\begin{array}{cc}\cos \psi / 2 & \sin \psi / 2 \\ -\sin \psi / 2 & \cos \psi / 2\end{array}\right)$ define $\lambda_{\psi} \in$ $\mathrm{GL}(\mathscr{C})$ by $\lambda_{\psi} v=\mu_{\psi} v \mu_{\psi}^{\prime}$. For every $z=(s, \psi, w) \in \mathbb{R}^{2} \times V$ then

$$
\theta_{z}(r, \varphi, v):=\left(r+s, \varphi+\psi, e^{s} \lambda_{\psi} v+w\right)
$$

defines an affine transformation $\theta_{z} \in \operatorname{Aut}(\widetilde{\mathscr{T}})$ and $\Theta:=\left\{\theta_{z}: z \in \mathbb{R}^{2} \times V\right\}$ is a Lie group acting freely and transitively on $\widetilde{\mathscr{T}}$. Clearly, $\Theta$ has many discrete subgroups $\Gamma$, each of which gives a CR-manifold $\widetilde{T} / \Gamma$ locally CR-equivalent to $\mathscr{T}$. Here we restrict our attention to the following one-parameter family $\left(\Gamma_{t}\right)_{t>0}$ of discrete subgroups: For every real $t>0$ put $\gamma_{t}:=\theta_{(0,2 \pi t, 0)}$, $\Gamma_{t}:=\left\{\gamma_{n t}: n \in \mathbb{Z}\right\}$ and $\mathscr{T}_{t}:=\widetilde{T} / \Gamma_{t}$. Then every $\mathscr{T}_{t}$ is isomorphic to $\mathscr{T}$ as real-analytic manifold while $\mathscr{T}_{1}$ is equivalent to $\mathscr{T}$ as CR-manifold. We will see later that actually the $\mathscr{T}_{t}$ are pairwise nonequivalent as CR-manifolds, compare Proposition 5.10. The family $\left(\mathscr{T}_{t}\right)_{t>0}$ may be considered as a CR-deformation family of $\mathscr{T} \cong \mathscr{T}_{1}$ : For $\mathbb{R}^{+}:=\{t \in \mathbb{R}: t>0\}$ consider the CR-manifold $\widetilde{\mathscr{T}} \times \mathbb{R}^{+}$, on which $\mathbb{Z}$ acts freely by $(z, t) \mapsto\left(\gamma_{n t} z, t\right), n \in \mathbb{Z}$. Then $\left(\widetilde{T} \times \mathbb{R}^{+}\right) / \mathbb{Z}$ is a real-analytic CR-manifold, and the canonical projection $\pi:\left(\widetilde{\mathscr{T}} \times \mathbb{R}^{+}\right) / \mathbb{Z} \rightarrow \mathbb{R}^{+}$is a CR-mapping whose fibers give the family $\left(\mathscr{T}_{t}\right)_{t>0}$.

5.5 Proposition. For every $t>0$ and every $a \in \mathscr{T}_{t}$ the cardinality $\sigma_{t}(a)$ of the set

$$
\Sigma_{t}(a):=\left\{z \in \mathscr{T}_{t}: f(z)=f(a) \text { for every continuous CR-function } f \text { on } \mathscr{T}_{t}\right\}
$$

is given by

$$
\sigma_{t}(a)= \begin{cases}p & t=p / q \text { for relatively prime integers } p, q>0 \\ \infty & \text { in all other cases. }\end{cases}
$$

Proof. The group $\Gamma:=\Gamma_{1} /\left(\Gamma_{1} \cap \Gamma_{t}\right)$ acts freely on $\mathscr{T}_{t}$, and by Proposition 5.2 every continuous CR-function on $\mathscr{T}_{t}$ is constant on $\Gamma$-orbits. In case $t$ irrational $\Gamma \cong \mathbb{Z}$ implies $\sigma_{t}(a)=\infty$. Therefore we may assume in the following that $t=p / q$ with relatively prime integers $p, q>0$. Since then $\Gamma$ has order $p$ we get $\sigma_{t}(a) \geq p$. On the other hand, the CR-manifold $\mathscr{T}_{1 / q}$ is separable with respect to real-analytic CR-functions. Indeed, the orbits of the finite subgroup $\Lambda:=\left\{\lambda_{2 \pi s}: s \in q^{-1} \mathbb{Z}\right\} \subset \mathrm{GL}(V) \subset \mathrm{GL}(E)$ are separated by the $\Lambda$-invariant holomorphic polynomials on $E$. Since we have a CR-covering map $\mathscr{T}_{p / q} \rightarrow \mathscr{T}_{1 / q}$ of degree $p$ this implies $\sigma_{t}(a) \leq p$.

It is not difficult to see that the centralizer of $\gamma_{t}$ in Aut $(\widetilde{\mathscr{T}})$ acts transitively on $\widetilde{\mathscr{T}}$ if and only if $t \in \mathbb{N}$. As a consequence, $\mathscr{T}_{t}$ is homogeneous as CR-manifold if and only if $t$ is an integer. In any case, the centralizer of $\gamma_{t}$ contains the 1-parameter subgroup $\left\{\gamma_{s}: s \in \mathbb{R}\right\}$ of Aut $(\widetilde{T})$, which therefore also acts on $\mathscr{T}$.

5.7 Proposition. For every irrational $t>0$ the following properties hold:

(i) For every $a \in \mathscr{T}_{t}$, the set $\Sigma_{t}(a)$ defined in (5.6) is the circle $\Sigma_{t}(a)=\left\{\gamma_{s}(a): 0 \leq s<t\right\}$.

(ii) There exists a closed hypersurface $\mathscr{N}_{t} \subset \mathscr{T}_{t}$ such that every continuous CR-function on $\mathscr{T}_{t}$ is real-analytic on the complement $\mathscr{T}_{t} \backslash \mathscr{N}_{t}$.

(iii) There exists a continuous $C R$-function on $\mathscr{T}_{t}$ which is not real-analytic. 
Proof. Fix a continuous CR-function $f$ on $\mathscr{T}_{t}$ and denote by $\mu: \widetilde{\mathscr{T}} \rightarrow \mathscr{T}, \mu_{t}: \widetilde{\mathscr{T}} \rightarrow \mathscr{T}_{t}$ the canonical projections. By Proposition 5.2 there exists a holomorphic function $h$ on $\mathscr{H} \cup \mathscr{T}$ with $f \circ \mu_{t}=h \circ \mu$. Since, for every $a \in \mathscr{T}_{t}$, the function $f$ is constant on a dense subset of the circle $\left\{\gamma_{s}(a): s \in \mathbb{R}\right\}$ we get $\Sigma_{t}(a) \subset\left\{\gamma_{s}(a): s \in \mathbb{R}\right\}$. This implies that $f \circ \mu_{t}$ is $\gamma_{s}$-invariant for every $s \in \mathbb{R}$ and hence that $h$ is invariant under $\mathrm{SO}(2)$ acting on $E$ as in (3.2). Now the proposition follows from the next lemmata 5.8 and 5.9, which may be of independent interest. The inclusion $\left\{\gamma_{s}(a): s \in \mathbb{R}\right\} \subset \Sigma_{t}(a)$ follows from the fact that the mapping $\varphi$ defined in Lemma 5.8 separates the $\mathrm{SO}(2)$-orbits in $\mathscr{T}$.

5.8 Lemma. Consider in $\mathscr{T}$ the hypersurface

$$
\mathscr{N}:=\{c+i(r c+s e): c \in \mathscr{C} \text { and } r, s \in \mathbb{R}\}, \quad e \text { the unit matrix, }
$$

and denote by $\mathscr{W}:=\mathscr{T} \backslash \mathscr{N}$ its complement. Let $\operatorname{tr}$ be the normalized trace on $E$ (i.e. $\operatorname{tr}(e)=1)$ and define the $\mathrm{SO}(2)$-invariant holomorphic mapping $\varphi: E \rightarrow \mathbb{C}^{2}$ by $\varphi(z):=\left(\operatorname{tr}(z) \operatorname{tr}\left(z^{2}\right)-\operatorname{tr}(z)^{2}\right)$. Then

$$
\begin{aligned}
\varphi(\mathscr{W}) & =\left\{\left(x_{1}+i y_{1}, x_{2}+i y_{2}\right) \in \mathbb{C}^{2}: x_{1}>0, x_{2}<x_{1}^{2}-\left(2 x_{1}\right)^{-2} y_{2}^{2}\right\} \\
\varphi(\mathscr{N}) & =\left\{\left(x_{1}+i y_{1}, x_{2}+i y_{2}\right) \in \mathbb{C}^{2}: x_{1}>0, x_{2}=x_{1}^{2}-\left(2 x_{1}\right)^{-2} y_{2}^{2}\right\}
\end{aligned}
$$

and to every continuous $\mathrm{SO}(2)$-invariant $C R$-function $h$ on $\mathscr{T}$ there exists a unique continuous function $f$ on $\varphi(\mathscr{T})$ with $h=f \circ \varphi$ such that the restriction of $f$ to the interior $\varphi(\mathscr{W})$ is holomorphic. In particular, every continuous $\mathrm{SO}(2)$-invariant $C R$-function on $\mathscr{T}$ is real-analytic on the dense domain $\mathscr{W} \subset \mathscr{T}$.

Proof. The expressions for $\varphi(\mathscr{W})$ and $\varphi(\mathscr{N})$ are checked by direct computation. Every continuous $\mathrm{SO}(2)$-invariant function $h$ on $\mathscr{T}$ is of the form $h=f \circ \varphi$ with $f$ a continuous function on $\varphi(\mathscr{T})$. In case $h$ is $\mathrm{CR}$ in addition, $h$ admits an extension to an $\mathrm{SO}(2)$-invariant holomorphic function on $\mathscr{H}$. This implies that $f$ is holomorphic on $\varphi(\mathscr{W})=\varphi(\mathscr{H})$.

For the proof of 5.7.(iii) it would be enough to find directly a continuous function $g$ on $\varphi(\mathscr{T})$ such that the pull-back $f:=g \circ \varphi$ is CR but not real-analytic on $\mathscr{T}$. Instead, we use invariant integration for the explicit determination of such an $f$.

5.9 Lemma. There exists a continuous $\mathrm{SO}(2)$-invariant function on $\mathscr{T}$ that is not real-analytic.

Proof. Let $\sqrt{s}$ be the unique continuous branch of the square root on $H:=\{s \in \mathbb{C}: \operatorname{Re}(s) \geq 0)\}$ with $\sqrt{1}=1$. Then $\lambda(z)=z_{11}$ defines a complex linear form $\lambda: E \rightarrow \mathbb{C}$ with $\lambda(\hat{\mathscr{T}}) \subset H$ for $\hat{\mathscr{T}}:=\mathscr{T} \cup \mathscr{H}$. The function $\varphi(z):=\sqrt{\lambda(z)}$ is continuous on $\hat{\mathscr{T}}$ and holomorphic on $\mathscr{H}$. For easier computation let us introduce on $E$ the coordinates $\left(w, z_{1}, z_{2}\right) \mapsto\left(\begin{array}{cc}w-z_{1} & z_{2} \\ z_{2} & w+z_{1}\end{array}\right)$, and then $\lambda\left(w, z_{1}, z_{2}\right)=w-z_{1}$. For every $t \in \mathbb{R}$ define $\gamma_{t} \in \mathrm{GL}(E)$ by

$$
\left(w, z_{1}, z_{2}\right) \longmapsto\left(w, z_{1} \cos t+z_{2} \sin t,-z_{1} \sin t+z_{2} \cos t\right) .
$$

Then

$$
h(z):=\int_{0}^{2 \pi} \varphi\left(\gamma_{t} z\right) d t
$$

defines a continuous SO(2)-invariant function $h$ on $\hat{\mathscr{T}}$ that is holomorphic on $\mathscr{H}$. As local uniform limit of holomorphic functions therefore the restriction $f:=\left.h\right|_{\mathscr{T}}$ is a continuous $\mathrm{SO}(2)$ invariant CR-function on $\mathscr{T}$. Define on the open unit disk $\Delta \subset \mathbb{C}$ the holomorphic function $g$ by

$$
g(s):=h(1, s, 0)=\int_{0}^{2 \pi} \sqrt{1-s \cos t} d t=\sum_{k=0}^{\infty}\left(\begin{array}{c}
1 / 2 \\
k
\end{array}\right) c_{k} s^{k}, \quad c_{k}:=\int_{0}^{2 \pi}(\cos t)^{k} d t,
$$


and denote by $R \geq 1$ the radius of convergence of the power series expansion. From the recursion $c_{0}=2 \pi, c_{1}=0$ and $c_{k}=\frac{k-1}{k} c_{k-2}$ for $k \geq 2$ we get $R=1$. Because of $(1, s, 0) \in \mathscr{T}$ for $|s|=1$ the function $g$ has a continuous extension to the closure $\bar{\Delta}$.

Now assume that $f$ is real-analytic on $\mathscr{T}$. Then $g$ would have a holomorphic extension to an open neighbourhood of $\bar{\Delta}$ in $\mathbb{C}$. This contradicts $R=1$, that is, $f$ cannot be real-analytic on $\mathscr{T}$.

5.10 Proposition. The manifolds $\mathscr{T}_{s}, \mathscr{T}_{t}$ are globally $C R$-equivalent if and only if $s=t$.

Proof. Assume that $\mathscr{T}_{s}, \mathscr{T}_{t}$ are globally CR-equivalent. Then there exists a transformation $g \in$ Aut $(\widetilde{T})$ with $g \Gamma_{s}=\Gamma_{t} g$, that is, $g \gamma_{s}=\gamma_{r} g$ with $r= \pm t$. In case $s \in \mathbb{N}$ the CR-manifold $\mathscr{T}_{s}$ is homogeneous. But then $\sigma_{t}(a)=\sigma_{s}(b)$ for all $a \in \mathscr{T}_{a}$ and $b \in \mathscr{T}_{s}$ implies $s=t$ as a consequence of Proposition 5.5. We therefore may assume $s, t \notin \mathbb{N}$. Inspecting the homomorphic image of the equation $g \gamma_{s}=\gamma_{r} g$ in Aut $(\mathscr{T})$ shows $g \in \Theta$ and thus $s=t$, see (5.4) for the definition of the group $\Theta$.

\section{The bounded realization}

Let again $E=\left\{z \in \mathbb{C}^{2 \times 2}: z^{\prime}=z\right\}$ be the linear space of all complex symmetric $2 \times 2$ matrices with $e=\mathbb{1}_{2} \in E$ the unit matrix. Put

$$
\begin{aligned}
& \mathscr{D}:=\{z \in E: e-z \bar{z} \text { positive definite }\} \\
& \mathscr{R}:=\{z \in E: \operatorname{det}(e-z \bar{z})=0, \operatorname{tr}(e-z \bar{z})>0\}=\{z \in \partial \mathscr{D}: z \bar{z} \neq e\} \\
& \mathscr{S}:=\{z \in E: z \text { unitary }\} \cong \mathrm{U}(2) / \mathrm{O}(2) \cong\left(S^{1} \times S^{2}\right) / \mathbb{Z}_{2},
\end{aligned}
$$

where the action of $\mathbb{Z}_{2}$ is generated by the antipodal map in each of the two spheres $S^{1}, S^{2}$. Then $\mathscr{D}$ is the open unit ball in $E$ with respect to the operator norm and is also called a Lie ball. The boundary $\partial \mathscr{D}$ decomposes into its smooth part $\mathscr{R}$ and the Shilov boundary $\mathscr{S}$, which is a totally real submanifold of $E$.

The CR-manifold $\mathscr{R}$ is fibered in its holomorphic arc components, which all are affinely equivalent to the open unit disk $\Delta \subset \mathbb{C}$, compare [16]. By definition, $A \subset \mathscr{R}$ is a holomorphic arc component of $\mathscr{R}$, if it is minimal with respect to the property: $A \neq \emptyset$, and $f(\Delta) \subset A$ for every holomorphic map $f: \Delta \rightarrow E$ with $f(\Delta) \subset \mathscr{R}$ and $f(\Delta) \cap A \neq \emptyset$. For instance, the set $A$ of all diagonal matrices $z \in E$ with $z_{11}=1>\left|z_{22}\right|$ is such an arc component, and every other is of the form $u A u^{\prime}$ with $u \in \mathrm{U}(2)$. This implies that the space of all arc components in $\mathscr{R}$ can be identified with $\mathrm{U}(2) /(\mathrm{O}(1) \times \mathrm{U}(1)) \cong \mathbb{P}_{3}(\mathbb{R})$. Since every $g \in \operatorname{Aut}(\mathscr{R})$ respects holomorphic arc components there is an Aut $(\mathscr{R})$-equivariant fiber bundle $\Xi: \mathscr{R} \rightarrow \mathbb{P}_{3}(\mathbb{R})$ with fibers the holomorphic arc components of $\mathscr{R}$.

It is well known that the Cayley transformation $z \mapsto(z-e)(z+e)^{-1}$ defines a biholomorphic map $\gamma: \mathscr{H} \rightarrow \mathscr{D}$ and also gives a CR-isomorphism $\mathscr{T} \stackrel{\widetilde{\leftrightarrows}}{\rightarrow}\{z \in \mathscr{R}: \operatorname{det}(e-z) \neq 0\}$. In fact, $E$ can be considered as Zariski-open subset of a nonsingular quadric $Z \subset \mathbb{P}_{4}(\mathbb{C})$ in such a way that $\gamma$ extends to a biholomorphic automorphism of $Z$, and then $\gamma^{2}(z)=-z^{-1}$ for all invertible $z \in E$. Also, there exists a unique antiholomorphic involution $\tau: Z \rightarrow Z$ with $\operatorname{Fix}(\tau)=\mathscr{S}$, and then

$$
\begin{aligned}
G: & =\{g \in \operatorname{Aut}(Z): g(\mathscr{D})=\mathscr{D}\}=\{g \in \operatorname{Aut}(Z): g(\mathscr{R})=\mathscr{R}\} \\
& =\{g \in \operatorname{Aut}(Z): \tau g \tau=g\}^{0} \cong \mathrm{SO}(2,3)^{0}
\end{aligned}
$$

acts transitively on $\mathscr{D}, \mathscr{R}, \mathscr{S}$. Via these actions the groups $G$, Aut $(\mathscr{D})$ and Aut $(\mathscr{R})$ are canonically isomorphic and hence are identified in the following. We also identify the Lie algebra

$$
\mathfrak{g}:=\mathfrak{a u t}(\mathscr{D})=\left\{\left(a+c z+z c^{\prime}-z \bar{a} z\right) \partial / \partial z: a \in E, c \in \mathfrak{u}(2)\right\}
$$


of $G$ with $\mathfrak{a u t}(\mathscr{R})=\mathfrak{h o l}(\mathscr{R})$, where $\mathfrak{u}(2) \subset \mathfrak{g l}(2, \mathbb{C})$ is the Lie subalgebra of all skew hermitian matrices.

The complexification $\mathfrak{l}=\mathfrak{g} \oplus i \mathfrak{g}$ has the decomposition

$$
\mathfrak{l}=\bigoplus_{\nu \in \mathbb{Z}^{2}} \mathfrak{l}^{v} \quad \text { with } \quad \mathfrak{l}^{\nu}:=\left\{\xi \in \mathfrak{l}:\left[\zeta_{j}, \xi\right]=\nu_{j} \xi \text { for } j=1,2\right\}
$$

with $\zeta_{j} \in \mathfrak{l}$ defined by (3.5) and (3.7) again being a choice of root vectors $\xi^{\nu} \in \mathfrak{l}^{\nu}$. But unlike to (3.6) the real form $\mathfrak{g}$ is embedded in a different way here:

$$
\mathfrak{g} \cap\left(\mathfrak{l}^{\nu}+\mathfrak{l}^{-\nu}\right)= \begin{cases}\mathbb{R}\left(\xi^{\nu}+\xi^{-\nu}\right) \oplus \mathbb{R}\left(i \xi^{\nu}-i \xi^{-\nu}\right) & \nu \neq 0 \\ \mathbb{R} i \zeta_{1} \oplus \mathbb{R} i \zeta_{2} & \text { otherwise }\end{cases}
$$

$\mathscr{R}$ has the remarkable property that every CR-equivalence between domains $\mathscr{U}, \mathscr{V}$ in $\mathscr{R}$ extends to a CR-automorphism of $\mathscr{R}$, compare [11]. In particular, Aut $(\mathscr{U}) \cong\{g \in G: g(\mathscr{U})=$ $\mathscr{U}\}$ and the groups $\operatorname{Aut}(\mathscr{U})$, Aut $(\mathscr{V})$ are conjugate in $\operatorname{Aut}(\mathscr{R})$ for any pair of CR-equivalent domains $\mathscr{U}, \mathscr{V} \subset \mathscr{R}$. Via the Cayley transformation $\mathscr{R}$ contains a copy of the CR-manifold $\mathscr{T}$ and hence may be considered as a CR-extension of $\mathscr{T}$. We may ask whether $\mathscr{R}$ is maximal with respect to $\mathrm{CR}$-extensions, that is, whether $\mathscr{R}$ can be a proper domain in some other connected CR-manifold. A partial answer is given by the following result.

6.3 Proposition. Let $M$ be a connected, locally homogeneous $C R$-manifold and let $D \subset M$ be a domain that is $C R$-isomorphic to a covering of $\mathscr{R}$. Then $D=M$.

Proof. Assume to the contrary $D \neq M$ and fix a boundary point $c$ of $D$ in $M$. Since $M$ is locally homogeneous there exists a connected open neighbourhood $U \subset M$ of $c$ and a vector field $\eta \in \mathfrak{h o l}(U)$ having an integral curve $\gamma:[0,1] \rightarrow U$ with $\gamma(0)=c$ and $a:=\gamma(1) \in D$. Since $D$ is CR-equivalent to a covering of $\mathscr{R}$ there exists a vector field $\xi \in \mathfrak{h o l}(D)$ having the same germ as $\eta$ at $a$. Since $\xi$ is complete on $D$ there exists an integral curve $g: \mathbb{R} \rightarrow D$ of $\xi$ with $g(0)=a$. But then, for every $s \in[0,1]$, we have $g_{-s}=\gamma_{1-s} \in D$. In particular, $c=g_{-1} \in D$ gives a contradiction.

6.4 Proposition. Let $M$ be a homogeneous, connected, simply connected real-analytic CR-manifold which is locally $C R$-isomorphic to $\mathscr{R}$. Then $M$ is $C R$-equivalent to the universal covering $\widetilde{\mathscr{U}}$ of a homogeneous domain $\mathscr{U} \subset \mathscr{R}$.

Proof. For every $a \in M$ let $\mathcal{F}_{a}$ be the set of all germs at $a$ of CR-equivalences $U \rightarrow \mathscr{V}$, where $U$ is a an open neighbourhood of $a \in M$ and $\mathscr{V}$ is open in $\mathscr{R}$. Consider the disjoint union $\mathcal{F}$ of all $\mathcal{F}_{a}$, $a \in M$, in the usual way as sheaf over $M$ with sheaf projection $\mu: \mathcal{F} \rightarrow M$ and fix a connected component $N$ of $\mathcal{F}$. By assumption there is a connected Lie group $H$ of CR-automorphisms of $M$ acting transitively on $M$. This implies that $\mu: N \rightarrow \mathscr{M}$ is a covering map and hence bijective. The evaluation map $N \rightarrow \mathscr{R}$ defines a CR-covering map onto the image $\mathscr{U} \subset \mathscr{R}$. Since this map is $H$-equivariant, $\mathscr{U}$ is a homogeneous domain in $\mathscr{R}$.

Proposition 6.4 reduces the classification problem for CR-manifolds locally CR-isomorphic to $\mathscr{R}$ to the study of homogeneous domains in $\mathscr{R}$. But these are in 1-1-correspondence to $G$ homogeneous domains in $\mathbb{P}_{3}(\mathbb{R})$. More precisely, every homogeneous domain $\mathscr{U} \subset \mathscr{R}$ is the full $\Xi$-pre-image of $U:=\Xi(\mathscr{U})$. Indeed, fix an arbitrary holomorphic arc component $A$ of $\mathscr{R}$ with $A \cap \mathscr{U} \neq \emptyset$. Then the group $H:=\{g \in \operatorname{Aut}(\mathscr{U}): g(A)=A\}$ has the orbit $A \cap \mathscr{U}$ that is open in $A \cong \Delta$. Therefore $H$ acts transitively on $A$ implying the claim. As a consequence, $\mathscr{U} \rightarrow U$ is a fiber bundle with contractible fiber, implying that the homogeneous domain $\mathscr{U} \subset \mathscr{R}$ and its $\Xi$-image $U \subset \mathbb{P}_{3}(\mathbb{R})$ always have the same homotopy type. In particular, $\mathscr{R}$ has fundamental group $\mathbb{Z}_{2}$. 
For certain homogeneous domains in $\mathscr{R}$ the same argument as in the proof of Proposition 5.2 yields the following holomorphic extension property.

6.5 Proposition. Let $\mu: \widetilde{\mathscr{U}} \rightarrow \mathscr{U}$ be the universal covering of a homogeneous domain $\mathscr{U} \subset \mathscr{R}$ and assume that $\operatorname{Aut}(\mathscr{U}) \subset \operatorname{Aut}(\mathscr{R})$ acts transitively on $\mathscr{D}$. Then to every continuous CR-function $f$ on $\widetilde{\mathscr{U}}$ there exists a unique continuous function $h$ on $\mathscr{U} \cup \mathscr{D}$ with the following two properties.

(i) $f=h \circ \mu$,

(ii) $h$ is holomorphic on $\mathscr{D}$.

To get further examples of homogeneous domains in $\mathscr{R}$ write

$$
G_{Y}:=\{g \in G: g(Y)=Y\} \text { and } \mathfrak{g}_{Y}:=\left\{\xi \in \mathfrak{g}: \exp (\mathbb{R} \xi) \subset G_{Y}\right\}
$$

for every subset $Y \subset Z$. Then every subgroup $G_{a}, a \in \mathscr{S}$, has a unique open orbit in $\mathscr{R}$. In case $a=-e$ this orbit is just the image $\gamma(\mathscr{T}) \subset \mathscr{R}$ of the tube manifold $\mathscr{T}$. Also, for every holomorphic arc component $A$ of $\mathscr{R}$ the group $G_{A}$ has a unique open orbit in $\mathscr{R}$. Even the intersection $G_{A} \cap G_{a}$ has a unique open orbit in $\mathscr{R}$, provided $a \in \mathscr{S}$ is in the closure of $A$ with respect to $\partial \mathscr{D}$. All these groups act transitively on $\mathscr{D}$ and thus give homogeneous domains in $\mathscr{R}$ with the holomorphic extension property of Proposition 6.5. But there also exist homogeneous domains $\mathscr{U} \subset \mathscr{R}$ such that $\operatorname{Aut}(\mathscr{U})$ is not transitive on $\mathscr{D}$ :

6.6 Example. Let $F \subset E$ be the $\mathbb{C}$-linear subspace of all diagonal matrices. Then the intersection $F \cap \mathscr{D}$ is biholomorphically equivalent to the bidisk $\Delta^{2} \subset \mathbb{C}^{2}$ and $G_{F \cap \mathscr{D}}$ has Lie algebra

$$
\mathfrak{g}_{F \cap \mathscr{D}}:=\left\{\left(a+c z+z c^{\prime}-z \bar{a} z\right) \partial / \partial z: a \in F, c \in F \cap \mathfrak{u}(2)\right\} \cong \mathfrak{s l}(2, \mathbb{R}) \times \mathfrak{s l}(2, \mathbb{R}) .
$$

The corresponding open orbit $\mathscr{U} \subset \mathscr{R}$ has the following equivalent description, which may be of interest for itself: The group $H:=\operatorname{SL}(2, \mathbb{R}) \times \mathrm{SL}(2, \mathbb{R})$ acts on the complex manifold $X:=\operatorname{SL}(2, \mathbb{C})$ by $z \mapsto g z h^{-1}$. For every $\left(\begin{array}{ll}a & b \\ c & d\end{array}\right) \in X$ put $\delta(z):=\operatorname{det}(z+\bar{z})-2=2 \operatorname{Re}(a \bar{d}-b \bar{c})$. Then $\delta$ is an $H$-invariant function on $X$ with $\delta(X)=\mathbb{R}$. It is easily checked that multiplication by $j:=\left(\begin{array}{ll}0 & i \\ i & 0\end{array}\right)$ induces a biholomorphic automorphism of $X$ with $\delta(j z)=-\delta(z)$. The critical set of $\delta$ is $\{z \in X: \bar{z}= \pm z\}=\operatorname{SL}(2, \mathbb{R}) \cup j \cdot \operatorname{SL}(2, \mathbb{R})$. In particular, $\delta$ has critical values \pm 2 . For every $\varepsilon \in\{ \pm i, 0\}$ consider the orbit $M_{\varepsilon}:=H\left(\begin{array}{ll}1 & \varepsilon \\ 0 & 1\end{array}\right)$. The inversion $z \mapsto z^{-1}$ maps $M_{\varepsilon}$ to $M_{-\varepsilon}$ and $\overline{M_{i}} \cap \overline{M_{-i}}=M_{0}=\mathrm{SL}(2, \mathbb{R})$. It can be shown that $M_{i}$ is CR-equivalent to $\mathscr{U} \subset \mathscr{R}$. Furthermore, $M_{i}$ is diffeomorphic to $\mathscr{C} \times \mathrm{SL}(2, \mathbb{R})$ and hence has fundamental group $\mathbb{Z}^{2}$ (recall that the tube manifold $\mathscr{T}$ is diffeomorphic to $\mathscr{C} \times V$ ).

6.7 Example. The Lie group $\mathrm{SU}(2) /\{ \pm e\} \cong \mathrm{SO}(3)$ acts on $E$ by $z \mapsto g z g^{\prime}$. Choose an $\mathbb{R}$-linear subspace $W$ of $E$ that is invariant under this action, e.g. $W=\left\{z \in E: z_{11}-\bar{z}_{22}=z_{12}+\bar{z}_{12}=0\right\}$. Denote by $\tau: E \rightarrow E$ the unique conjugate linear involution fixing every point of $W$. Then $\tau(\mathscr{D})=\mathscr{D}$ and the intersection $W \cap \mathscr{D}$ is a euclidian ball in $W$. The group $G_{W \cap \mathscr{D}}=\{g \in G$ : $\tau g \tau=g\}$ has Lie algebra

$$
\mathfrak{g}_{W \cap \mathscr{D}}=\left\{\left(a+c z+z c^{\prime}-z \bar{a} z\right) \partial / \partial z: a \in W, c \in \mathfrak{s u}(2)\right\} \cong \mathfrak{s l}(2, \mathbb{C}) .
$$

Again, the corresponding open orbit $\mathscr{U} \subset \mathscr{R}$ has an equivalent realization: Let $X:=\mathrm{SL}(2, \mathbb{C})$ and consider $H:=\mathrm{SL}(2, \mathbb{C})$ as real Lie group. Then $H$ acts on $X$ by $z \mapsto g z g^{*}$, where $g^{*}$ is the conjugate transpose of $g$. Clearly, $z \mapsto-z$ commutes with the action of $H$ and hence permutes $H$-orbits. The function $\delta(z):=\operatorname{det}\left(z+z^{*}\right)-2=a \bar{d}+d \bar{a}-b \bar{b}-c \bar{c}$ on $X$ is $H$-invariant with critical values \pm 2 and critical set $\left\{z \in X: z^{*}= \pm z\right\}$. This set is the union of three totally real 
orbits, more precisely:

Critical value 2: There are the two critical orbits $N:=\left\{h \in X: h^{*}=h>0\right\}$ and $-N=\{h \in X$ : $\left.h^{*}=h<0\right\}$. A diffeomorphism $\left\{h=h^{*} \in \mathbb{C}^{2 \times 2}: \operatorname{tr}(h)=0\right\} \rightarrow N$ is given by the exponential mapping. By elementary calculus it is seen that $\delta$ attains a local maximum at every point of $\pm N$. As a consequence, $N$ is the only $H$-orbit in $X$ having $N$ in its closure (and the same with $-N)$.

Critical value -2 : The only critical orbit is $N:=\left\{i h: h=h^{*} \in \mathbb{C}^{2 \times 2}\right.$, $\left.\operatorname{det}(h)=-1\right\}$. The hypersurface orbit $M:=H\left(\begin{array}{ll}1 & i \\ i & 0\end{array}\right)$ has $N$ in its closure. It can be shown that $M$ is CR-equivalent to $\mathscr{U}$.

\section{Some final remarks.}

The idea of proof in Propositions 5.2 and 6.5 can also be applied in higher dimensions: For $n \geq 3$ fixed let $V \subset \mathbb{R}^{n \times n}$ be the subspace of all symmetric $n \times n$-matrices and $E:=V \oplus i V \subset \mathbb{C}^{n \times n}$ its complexification. For all $p, q \geq 0$ with $k:=n-(p+q) \geq 0$ denote by $\mathscr{C}_{p, q} \subset V$ the cone of all real symmetric $n \times n$-matrices of type $(p, q)$, that is, having $p$ positive and $q$ negative eigenvalues. The group $\mathrm{GL}(n, \mathbb{R})$ acts on $V$ by $x \mapsto g x g^{\prime}$, and the cones $\mathscr{C}_{p, q}$ are the corresponding orbits. Because of $\mathscr{C}_{q, p}=-\mathscr{C}_{p, q}$ we may restrict our attention to the special case $p \geq q$. The cone $\Omega:=\mathscr{C}_{n, 0}$ is convex open, and $\mathscr{H}:=\Omega \oplus i V \subset E$ is a symmetric tube domain (Siegel's upper half plane of rank $n$ up to the factor $i$ ). The group Aut $(\mathscr{H})$ consists of all biholomorphic transformations (3.3) with $\left(\begin{array}{ll}a & b \\ c & d\end{array}\right)$ in the $\operatorname{symplectic} \operatorname{subgroup} \operatorname{Sp}(n, \mathbb{R}) \subset \operatorname{SL}(2 n, \mathbb{R})$, compare [12] p. 345.

In analogy to our setting in Section 3 with $n=2$ we denote for every $p, q$ by $\mathscr{T}_{p, q}:=$ $\mathscr{C}_{p, q} \oplus i V$ the tube manifold over the cone $\mathscr{C}_{p, q}$. Then $\mathscr{T}_{p, q}$ is open precisely if $k=0$ and is closed if $k=n$. In all other cases $\mathscr{T}_{p, q}$ is a 2-nondegenerate CR-manifold and Aut $\left(\mathscr{T}_{p, q}\right)$ consists of all transformations $(3.2)$ (with $\mathrm{GL}(2, \mathbb{R})$ replaced by $\mathrm{GL}(n, \mathbb{R})$, compare [10]). In [10] also the following holomorphic extension property has been shown: In case $p q \neq 0$ every continuous $C R$ function on $\mathscr{T}_{p, q}$ has a holomorphic extension to all of $E$. In case $p>0$ every continuous $C R$-function on $\mathscr{T}_{p, 0}$ has a holomorphic extension to $\mathscr{H}$ that is continuous up to $\mathscr{T}_{p, 0} \subset \overline{\mathscr{H}}$ in a certain sense.

Note that every $\mathscr{T}_{p, q}$ is homogeneous under $\operatorname{GL}(n, \mathbb{R})^{0} \ltimes V$. Applying the decomposition theorem of Mostow one deduces that $\mathscr{T}_{p, q}$ is a bundle with contractible fibers over the homogeneous space $\mathrm{SO}(n) / S(\mathrm{O}(p) \times \mathrm{O}(q) \times \mathrm{O}(k))$. Explicit computation shows that $\mathrm{SO}(n)$ is a maximal compact subgroup of $\mathrm{GL}(n, \mathbb{R})^{0}$ and that for the diagonal matrix $a:=\mathbb{1}_{p} \times-\mathbb{1}_{q} \times 0_{k} \in \mathscr{T}_{p, q}$ the subgroup $S(\mathrm{O}(p) \times \mathrm{O}(q) \times \mathrm{O}(k)) \subset \mathrm{SO}(n)$ is maximal compact in the isotropy subgroup $\left\{g \in \mathrm{GL}\left(n, \mathbb{R}^{n}\right)^{0}: g a g^{\prime}=a\right\}$. As a consequence, employing the long exact homotopy sequence, we determine the fundamental group of $\mathscr{T}_{p, q}$ for $n \geq 3$ and $0<p+q<n$ as

$$
\pi_{1}\left(\mathscr{T}_{p, q}\right)= \begin{cases}Q_{8} & n=3, p=q=1 \\ \mathbb{Z}_{2} \oplus \mathbb{Z}_{2} & n>3, p>0, q>0 \\ \mathbb{Z}_{2} & \text { otherwise }\end{cases}
$$

where $Q_{8}$ is the quaternion group of order 8 .

For the universal covering $\mu: \widetilde{\mathscr{T}}_{p, q} \rightarrow \mathscr{T}_{p, q}$ of $\mathscr{T}_{p, q}$ we get the following higher dimensional analog of Proposition 5.2.

7.1 Proposition. In case $0<p+q<n$ every continuous $C R$-function on $\widetilde{\mathscr{T}}_{p, q}$ is constant on $\mu$-fibers.

Proof. Let $H$ be the group of all transformations $z \mapsto g z g^{\prime}+i v$ on $E$ with $g \in \mathrm{GL}(n, \mathbb{R})$ and $v \in V$. To begin with assume first that $p \cdot q>0$ and fix a continuous CR-function $f$ on $\widetilde{\mathscr{T}}_{p, q}$. 
Let $U \subset E$ be the smallest open $H$-invariant subset containing $\mathscr{T}_{p, q}$, that is, $U$ is the union of all $\mathscr{T}_{p^{\prime}, q^{\prime}}$ with $p^{\prime} \geq p$ and $q^{\prime} \geq q$. Denote by $\mathcal{O}$ the sheaf over $U$ of all germs of holomorphic functions. Since for every $a \in \mathscr{T}_{p, q}$ the Levi cone at $a$ spans the full normal space to $\mathscr{T}_{p, q}$ in $E$, for every $x \in \widetilde{\mathscr{T}}_{p, q}$ the function $f$ induces a germ $f_{x} \in \mathcal{O}_{\mu(x)}$. Denote by $\mathcal{F}$ the connected component of $\mathcal{O}$ containing all $f_{x}$ with $x \in \widetilde{\mathscr{T}}_{p, q}$. As in the proof of Proposition 5.2 it follows that $\mathcal{F}$ is a covering of $U$. As a consequence of the following Lemma 7.2 every loop in $\mathscr{T}_{p, q}$ is zero-homotopic in $U$. Therefore $\left\{f_{x}: x \in \widetilde{\mathscr{T}}_{p, q}\right\}$ is a trivial covering of $\mathscr{T}_{p, q}$, that is, $f=g \circ \mu$ for some CR-function on $\mathscr{T}_{p, q}$.

It remains to consider the case $q=0$. But then $\mathscr{T}_{p, 0} \cup \mathscr{H}$ is simply connected and the claim follows in the same way as in the proof of Proposition 5.2 (for every open subset $V$ of $\mathscr{T}_{p, 0} \cup \mathscr{H}$ a function $g$ on $V$ should be called holomorphic if its restriction to $V \cap \mathscr{H}$ is holomorphic in the usual sense and if for every $a \in V \cap \mathscr{T}_{p, q}$ there is a neighbourhood $W$ of $a$ in $\mathscr{T}_{p, q}$ and a wedge $\Gamma$ with edge $W$ such that the restriction $g_{\mid \Gamma}$ extends continuously to $W$ ).

7.2 Lemma. For every $p, q$ with $p+q<n$ the canonical injection $\mathscr{C}_{p, q} \hookrightarrow \mathscr{C}_{p+1, q} \cup \mathscr{C}_{p, q} \cup \mathscr{C}_{p, q+1}$ induces the trivial homomorphism between the corresponding fundamental groups.

Proof. Consider the 1-parameter subgroup of $\mathrm{SO}(n)$ given by

$$
g_{t}:=\left(\begin{array}{ccc}
\cos t & -\sin t & \\
\sin t & \cos t & \\
& & \mathbb{1}_{n-2}
\end{array}\right)
$$

for all $t \in \mathbb{R}$. Furthermore fix diagonal matrices $a, b \in \mathscr{C}_{p, q}$ with $a_{11}=1=-b_{11}$ and $a_{22}=$ $b_{22}=0$. Then the loops $\gamma, \sigma:[0, \pi] \rightarrow \mathscr{C}_{p, q}$ defined by $\gamma(t):=g_{t} a g_{t}^{\prime}$ and $\sigma(t):=g_{t} b g_{t}^{\prime}$ generate the group $\pi_{1}\left(\mathscr{C}_{p, q}\right)$. For every $s \in \mathbb{R}$ let $a(s), b(s)$ be the matrices obtained from $a, b$ by putting $a(s)_{22}=b(s)_{22}=s$ and leaving all other entries unchanged. Then $a(0)=a$ and $a(s) \in \mathscr{C}_{p+1, q}$ for all $s>0$. But then the loops $\gamma_{s}:[0, \pi] \rightarrow \mathscr{C}_{p, q} \cup \mathscr{C}_{p+1, q}, s \geq 0$, defined by $\gamma_{s}(t):=g_{t} a(s) g_{t}^{\prime}$ give a homotopy from $\gamma$ to the constant loop $\gamma_{1}$. In the same way the loops $\sigma_{s}:[0, \pi] \rightarrow \mathscr{C}_{p, q} \cup \mathscr{C}_{p, q+1}$, $s \leq 0$, defined by $\sigma_{s}(t):=g_{t} b(s) g_{t}^{\prime}$ give a homotopy from $\sigma$ to the constant loop $\sigma_{-1}$. domain

It is well known that the tube domain $\mathscr{H}$ has a canonical realization as bounded symmetric

$$
\mathscr{D}:=\left\{z \in E: \mathbb{1}_{n}-z \bar{z} \text { positive definite }\right\}
$$

given by the Cayley transformation $\gamma: z \mapsto\left(z-\mathbb{1}_{n}\right)\left(z+\mathbb{1}_{n}\right)^{-1}$. The boundary $\partial \mathscr{D}$ is the union of the CR-submanifolds of $E$

$$
\mathscr{R}_{p, 0}:=\left\{z \in E:\left(\mathbb{1}_{n}-z \bar{z}\right) \in \mathscr{C}_{p, 0}\right\}, \quad 0 \leq p<n .
$$

The group $\operatorname{Aut}(\mathscr{D})$ acts transitively on each $\mathscr{R}_{p, 0}$ and can be identified with $\operatorname{Aut}\left(\mathscr{R}_{p, 0}\right)$ this way for every $p>0$, see [11]. The Cayley transformation $\gamma$ gives a CR-isomorphism from $\mathscr{T}_{p, 0}$ to the dense domain $\left\{z \in \mathscr{R}_{p, 0}: \operatorname{det}\left(\mathbb{1}_{n}-z \bar{z}\right) \neq 0\right\}$ in $\mathscr{R}_{p, 0}$. Furthermore, the manifold $\mathscr{R}_{p, 0}$ is an Aut $(\mathscr{H})$-equivariant fiber bundle over $\mathrm{U}(n) /(\mathrm{O}(n-p) \times \mathrm{U}(p))$ with fibers the holomorphic arc components. In particular, $\pi_{1}\left(\mathscr{R}_{p, 0}\right)=\mathbb{Z}_{2}$ for $p<n$. As in the proof of Proposition 5.2 it is shown for every $p>0$ that every continuous CR-function on the universal covering $\mu: \widetilde{\mathscr{R}}_{p, 0} \rightarrow \mathscr{R}_{p, 0}$ is constant on $\mu$-fibers.

Every $\mathscr{T}_{p, 0}$ has the following local description in terms of normalized coordinates generalizing (4.17): For $k:=n-p$ put

$$
W:=\left\{w \in \mathbb{C}^{k \times k}: w^{\prime}=w\right\}, \quad E_{1}:=\mathbb{C}^{p \times k}, \quad E_{2}:=\left\{z_{2} \in \mathbb{C}^{p \times p}: z_{2}^{\prime}=z_{2}\right\}
$$


and denote by $\mathscr{M}$ in $U:=\left\{\left(w, z_{1}, z_{2}\right) \in W \times E_{1} \times E_{2}: \mathbb{1}_{p}-z_{2} \bar{z}_{2}>0\right\}$ the CR-submanifold given by the matrix equation

$$
w+\bar{w}=x+\bar{x} \quad \text { with } \quad x:=\bar{z}_{1}^{\prime}\left(\mathbb{1}_{p}-z_{2} \bar{z}_{2}\right)^{-1}\left(z_{1}+z_{2} \bar{z}_{1}\right) .
$$

Then

$$
\left(w, z_{1}, z_{2}\right) \longmapsto\left(\begin{array}{cc}
w+z_{1}^{\prime} y z_{1} & \sqrt{2} z_{1}^{\prime} y \\
\sqrt{2} y z_{1} & \left(\mathbb{1}_{p}-z_{2}\right) y
\end{array}\right) \quad \text { with } \quad y:=\left(\mathbb{1}_{p}+z_{2}\right)^{-1}
$$

defines a biholomorphic mapping $\varphi$ from $U$ to a domain in $E$ such that $\varphi(\mathscr{M})$ is open in $\mathscr{T}_{p, 0}$. This follows from the realization of $\mathscr{H}$ as Siegel domain of the third kind in terms of the partial Cayley transformation (7.4), compare [15] and [13] p. 10.7.

Due to the coincidence $\mathfrak{s p}(2, \mathbb{R}) \cong \mathfrak{s o}(2,3)$ between symplectic and orthogonal groups in low dimensions there are two ways to generalize the tube $\mathscr{T}$ over the light cone $\mathscr{C} \subset \mathbb{R}^{3}$ to higher dimensions: The first possibility in terms of symmetric $n \times n$-matrices and the symplectic group has been explicitly described above. The corresponding tube manifolds $\mathscr{T}_{p, q}, 0<p+q<n$, are all not simply connected and hence provide nontrivial examples for Proposition 7.1. Let us close with a description of the second way associated with orthogonal groups: For fixed $n \geq 2$ let

$$
\langle z \mid w\rangle:=z_{1} w_{1}+\ldots+z_{n} w_{n}
$$

be the standard symmetric bilinear form and $z \mapsto \bar{z}=\left(\bar{z}_{1}, \ldots, \bar{z}_{n}\right)$ the standard conjugation on $\mathbb{C}^{n}$. Denote by

$$
\mathscr{C}:=\left\{(t, x) \in \mathbb{R}^{2} \times \mathbb{R}^{n}: t_{1} t_{2}=\langle x \mid x\rangle, t_{1}+t_{2}>0\right\}
$$

the future light cone in $(n+2)$-dimensional space time and by $\mathscr{T}:=\mathscr{C} \oplus i \mathbb{R}^{n+2}$ the corresponding tube manifold over $\mathscr{C}$. Then $\mathscr{T}$ is a simply connected, 2-nondegenerate homogeneous CR-manifold with $\mathfrak{h o l}(\mathscr{T}, a) \cong \mathfrak{s o}(2, n+2)$ for all $a \in \mathscr{T}$, see [11] for the last statement. Then the hypersurface $\mathscr{M}$, given in $U:=\left\{\left(w, z_{1}, z_{2}\right) \in \mathbb{C} \times \mathbb{C}^{n} \times \mathbb{C}:\left|z_{2}\right|<1\right\}$ by the equation

$$
w+\bar{w}=\left(2\left\langle z_{1} \mid \bar{z}_{1}\right\rangle+\left\langle z_{1} \mid z_{1}\right\rangle \bar{z}_{2}+\left\langle\bar{z}_{1} \mid \bar{z}_{1}\right\rangle z_{2}\right)\left(1-z_{2} \bar{z}_{2}\right)^{-1},
$$

is CR-equivalent to a domain in $\mathscr{T}$. As in (4.17) an explicit equivalence is obtained by

$$
\left(w, z_{1}, z_{2}\right) \mapsto\left(1+z_{2}\right)^{-1}\left(w+w z_{2}+\left\langle z_{1} \mid z_{1}\right\rangle, 1-z_{2}, \sqrt{2} z_{1}\right)
$$

Acknowledgment: The authors wish to thank D. Zaitsev for helpful discussions.

\section{References}

1. Azad, H., Huckleberry, A., Richthofer, W.: Homogeneous CR-manifolds. J. Reine Angew. Math. 358 (1985), 125-154.

2. Baouendi, M.S., Ebenfelt, P., Rothschild, L.P.: Real Submanifolds in Complex Spaces and Their Mappings. Princeton Math. Series 47, Princeton Univ. Press, 1998.

3. Boggess, A.: CR Manifolds and the Tangential Cauchy-Riemann Complex. Studies in Advanced Mathematics. CRC Press. Boca Raton Ann Arbor Boston London 1991. 
4. Burns, D., Shnider, S.: Spherical hypersurfaces in complex manifolds. Invent. Math. 33 (1976), 223-246.

5. Chern, S.S., Moser, J.K.: Real hypersurfaces in complex manifolds. Acta. Math. 133 (1974), 219-271.

6. Ebenfelt, P.: Normal Forms and Biholomorphic Equivalence of Real Hypersurfaces in $\mathbb{C}^{3}$. Indiana J. Math. 47 (1998), 311-366.

7. Ebenfelt, P.: Uniformly Levi degenerate CR manifolds: the 5-dimensional case. Duke Math. J. 110 (2001), 37-80.

8. Ershova, A.E.: Automorphisms of 2-Nondegenerate Hypersurfaces in $\mathbb{C}^{3}$. Math. notes 69 (2001), 188-195.

9. Gaussier, H., Merker, J.: A new example of a uniformly Levi degenerate hypersurface in $\mathbb{C}^{3}$. Ark. Mat. 41 (2003), 85-94.

10. Kaup, W.: On the holomorphic structure of $G$-orbits in compact hermitian symmetric spaces. Math. Z. 249 (2005), 797-816.

11. Kaup, W., Zaitsev, D.: On the local CR-structure of Levi-degenerate group orbits in compact Hermitian symmetric spaces. J. Eur. Math. Soc., to appear

12. Klingen, H.: Diskontinuierliche Gruppen in symmetrischen Räumen. Math. Ann. 129 (1955), 345369.

13. Loos, O.: Bounded symmetric domains and Jordan pairs. Mathematical Lectures. Irvine: University of California at Irvine 1977.

14. Palais, R.S.: A global formulation of the Lie theory of transformation groups. Mem. AMS 1957.

15. Piatetsky-Chapiro, I.I.: Géométrie des domaines classiques et théorie des fonctions automorphes. Dunod, Paris 1966.

16. Wolf, A.J.: Fine Structure of Hermitian Symmetric Spaces. Symmetric spaces (Short Courses, Washington Univ., St. Louis, Mo., 1969-1970), pp. 271-357. Pure and App. Math., Vol. 8, Dekker, New York, 1972.

Mathematisches Institut, Universität Tübingen, Auf der Morgenstelle 10, 72076 Tübingen, Germany (e-mails: kaup@uni-tuebingen.de, fels@uni-tuebingen.de) 